\title{
Gene Erosion Can Lead to Gain-of-Function Alleles That Contribute to Bacterial Fitness
}

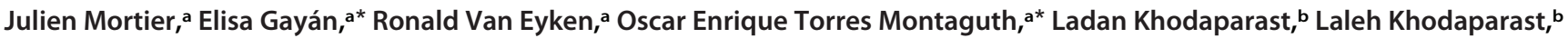 \\ Bert Houben, ${ }^{b}$ Sebastien Carpentier, ${ }^{c}$ Frederic Rousseau, ${ }^{\text {b Joost Schymkowitz, }}{ }^{\text {b }}$ (D) Abram Aertsen ${ }^{a}$
}

aKU Leuven, Department of Microbial and Molecular Systems, Leuven, Belgium
'KU Leuven, Department of Cellular and Molecular Medicine, Leuven, Belgium
cKU Leuven, SYBIOMA: Facility for Systems Biology Mass Spectrometry, Leuven, Belgium

Julien Mortier and Elisa Gayán contributed equally to the work. Author order was determined by increasing seniority.

ABSTRACT Despite our extensive knowledge of the genetic regulation of heat shock proteins (HSPs), the evolutionary routes that allow bacteria to adaptively tune their HSP levels and corresponding proteostatic robustness have been explored less. In this report, directed evolution experiments using the Escherichia coli model system unexpectedly revealed that seemingly random single mutations in its thaA gene can confer significant heat resistance. Closer examination, however, indicated that these mutations create folding-deficient and aggregation-prone TnaA variants that in turn can endogenously and preemptively trigger HSP expression to cause heat resistance. These findings, importantly, demonstrate that even erosive mutations with disruptive effects on protein structure and functionality can still yield true gain-of-function alleles with a selective advantage in adaptive evolution.

KEYWORDS evolution, genetics, heat resistance, protein aggregates

(1) roteotoxic stresses are amply encountered in both natural and manmade environments (1-6) and therefore constitute a primordial concern for bacteria. Indeed, conditions such as heat, reactive oxygen species, low $\mathrm{pH}$, and the presence of antimicrobial compounds may cause the misfolding and aggregation of nascent polypeptides and/or existing proteins in the cell (7). To counter these proteotoxic stresses and ensure proper protein homeostasis (proteostasis), bacteria have developed an elaborate protein quality control network that aims to refold or degrade improperly folded and aggregating polypeptides through the action of chaperones and proteases (e.g., DnaK, DnaJ, HtpG, GroEL, Lon, etc.), which are collectively referred to as heat shock proteins (HSPs) (8-10).

In the Escherichia coli model system, the level of HSP expression is mainly controlled by the RpoH $\left(\sigma^{32}\right)$ sigma factor, which is itself subjected to extensive transcriptional and translational regulation (11). While the $r p o H$ mRNA has a built-in thermosensor that unblocks the ribosome binding site at elevated temperatures, the $\mathrm{RpoH}$ protein becomes sequestered by DnaK and GroEL and targeted for degradation by the FtsH protease. When these sequestering chaperones are titrated away by damaged proteins that emerge when the cell encounters proteotoxic stress, $\mathrm{RpoH}$ will be released to occupy the RNA polymerase and raise HSP expression (11-14).

Despite extensive knowledge of the genetic regulation of HSPs, the accessible evolutionary routes that allow bacteria to adaptively tune their HSP levels and that account for corresponding intraspecies differences in proteostatic robustness have been explored less. In this context, some $E$. coli strains were shown to have extended their complement of available HSPs by the lateral acquisition of so-called transmissible
Citation Mortier J, Gayán E, Van Eyken R, Torres Montaguth OE, Khodaparast L, Khodaparast L, Houben B, Carpentier S, Rousseau F, Schymkowitz J, Aertsen A. 2021. Gene erosion can lead to gain-of-function alleles that contribute to bacterial fitness. mBio 12:e0112921. https://doi.org/10.1128/mBio.01129-21. Invited Editor Kristina Jonas, Stockholm University

Editor Lotte Søgaard-Andersen, Max Planck Institute for Terrestrial Microbiology Copyright $\odot 2021$ Mortier et al. This is an open-access article distributed under the terms of the Creative Commons Attribution 4.0 International license.

Address correspondence to Abram Aertsen abram.aertsen@kuleuven.be.

* Present address: Elisa Gayán, University of Zaragoza-CITA, Laboratory of Food Technology, Department of Animal Production and Food Science, AgriFood Institute of Aragon (IA2), Faculty of Veterinary, Zaragoza, Spain; Oscar Enrique Torres Montaguth, DNAProtein Interactions Unit, School of Biochemistry, Faculty of Life Sciences, University of Bristol, Bristol, United Kingdom.

Received 30 April 2021

Accepted 4 June 2021

Published 6 July 2021 
loci for protein quality control, thereby massively boosting their resistance against heat and oxidative stress and raising concerns for their increased survival in medical and industrial settings $(15,16)$. However, the prevalence of these transmissible islands is rather modest (17), and likely, more subtle evolutionary routes for modulating HSP levels exist as well.

In this study, we provide evidence for such an unanticipated adaptive evolutionary route in which $E$. coli mitigates the impact of proteotoxic stress by mutationally sacrificing the folding fidelity of a single, nonessential, and transiently expressed protein in order to preemptively activate its heat shock response. As such, we demonstrate that erosive mutations with disruptive effects on protein structure can serve as true gain-offunction mutations adaptively raising HSP levels.

\section{RESULTS}

Adaptation to heat stress selects for mutants with altered PA management. During preliminary directed evolution experiments, we (i) found that an E. coli MG1655 $\Delta$ rpos mutant (i.e., deprived of its $\sigma^{\mathrm{S}}$ general stress response regulator) could readily complement its intrinsic hypersensitivity to heat (Fig. 1A), and (ii) serendipitously noticed through phase-contrast microscopy that some such independently evolved heat resistant MG1655 $\Delta$ rpoS mutants typically displayed cells bearing a highly refractive polar structure, reminiscent of a protein aggregate (PA) (Fig. 1B and C). To address these initial experimental observations more systematically, we subsequently heatcycled 17 independent lineages of E. coli MG1655 $\Delta$ rpoS ibpA-msfGFP (able to fluorescently report on the presence of intracellular PAs through the IbpA-msfGFP reporter [18]), and from each lineage retained one random heat-resistant clone (Fig. 2A). Interestingly, 14 out of these 17 independent heat-resistant mutants (all except H4, $\mathrm{H} 14$, and $\mathrm{H} 17$ ) displayed considerably higher fractions of PA-bearing cells (especially toward the early and/or late stationary phase of growth) compared to the parental strain and its two control-cycled derivatives (Fig. 2B and C), thereby consolidating our observation that adaptive evolution toward heat resistance can alter cellular PA management.

Heat resistance causally stems from gain-of-function TnaA variants. Surprisingly, whole-genome sequencing of the 17 independent heat-resistant mutants revealed that 13 of them harbored a mutation in the thaA gene (encoding the tryptophanase enzyme; Table 1). Out of these 13 thaA mutants, 11 stood out as having clearly altered cellular PA management, while $2(\mathrm{H} 4$ and $\mathrm{H} 17)$ were hardly distinguishable from the parental MG1655 $\Delta r p o S$ ibpA-msfGFP strain with regard to the IbpA-msfGFP PA reporter (Fig. 2B). Moreover, checking the thaA allele of some of the preliminary heatcycled mutants, and even of previously reported hydrostatic pressure-cycled mutants (19), revealed another 4 tnaA alleles (Table 1). Interestingly, most of these thaA alleles (14 out of 17) were found to be unique and incurred point mutations, premature stop codons, or indels (both in-frame or frameshifting) throughout the thaA open reading frame (Table 1). Moreover, while many tnaA mutants were partially or fully compromised in their tryptophanase activity (i.e., the ability to produce indole from tryptophan), some of them displayed no obvious loss-of-function signs (Table 1).

To infer causality between possible tnaA alterations and heat resistance, the wildtype tnaA allele (tnaA ${ }^{W T}$ ) of $E$. coli MG1655 $\Delta$ rpoS was replaced with either (i) tnaA $A^{\Delta 106}$ as one of the evolved alleles (coming from mutant MT3; Table 1), (ii) $\Delta$ tnaA (unable to produce the TnaA protein), or (iii) tna $A^{K 270 A}$ (producing a catalytically compromised TnaA variant [20]) and subsequently heat challenged (Fig. 3A). Interestingly, this revealed not only that the tnaA $A^{\Delta 106}$ allele itself was indeed causally sufficient to increase heat resistance of MG1655 $\Delta r p o S$ to the same level as seen in the original MT3 mutant, but also that the $\Delta t n a A$ and $t n a A^{K 270 A}$ alleles failed to affect this phenotype (Fig. $3 \mathrm{~A}$ ), despite sharing an impaired ability to produce indole with the tnaA $A^{\Delta 106}$ allele (Fig. $3 C$ ). Moreover, in contrast to the tna $A^{W T}, \Delta t n a A$, and tna $A^{K 270 A}$ alleles, implementation of the $t n a A^{\Delta 106}$ allele also caused MG1655 $\Delta r p o S$ to produce PAs to the same level as seen in MT3, indicating that expression of this variant directly or indirectly causes 
A

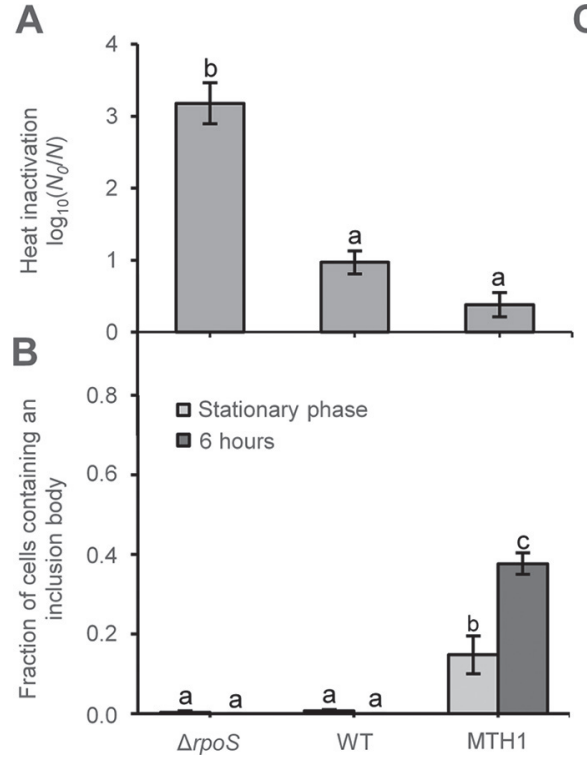

C

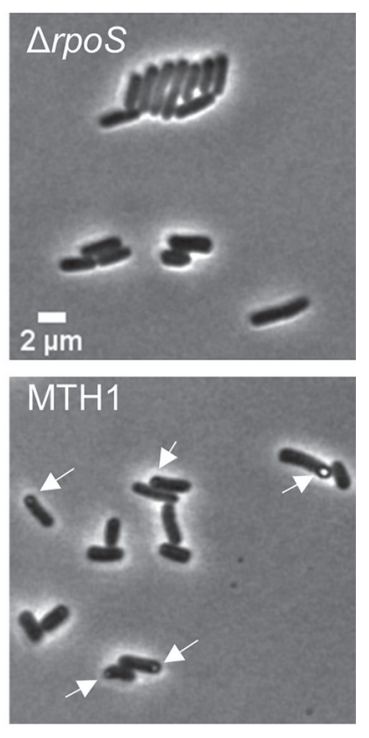

FIG 1 (A) Logarithmic reduction factor after exposure to heat $\left(55^{\circ} \mathrm{C}\right.$ for 15 min) of TSB-grown stationary-phase cultures of MG1655 wild-type (WT), MG1655 $\Delta$ rpoS, and a representative heatresistant mutant (MTH1) derived from MG1655 $\Delta$ rpoS by directed evolution (i.e., successive cycles of increasingly severe heat shocks with intermittent outgrowth to stationary phase in TSB). Different letters indicate statistically (Tukey HSD post hoc test, $P$ value $\leq 0.05$ ) significant differences among strains. (B) Fraction of cells that contain an inclusion body visible through phase-contrast microscopy for the indicated strains in TSB-grown stationary-phase populations and $6 \mathrm{~h}$ after reinoculating $1 / 100$ in fresh TSB. On average, 93.2 cells were observed per strain and condition per independent experiment, and all sample sizes were between 85 and 110 cells. Different letters indicate statistically significant differences (Tukey HSD post hoc test, $P$ value $\leq 0.05$ ) among different strains and time points. For panels $A$ and $B$, the displayed means were determined over three independent experiments, and the error bars indicate the standard error over these experiments. (C) Representative phase contrast images of MG1655 $\Delta$ rpoS (upper panel) and its evolved heat-resistant MTH1 mutant (lower panel) after stationary-phase growth in TSB. White arrows indicate inclusion bodies. Scale bar corresponds to $2 \mu \mathrm{m}$.

protein aggregation (Fig. 3B). As such, it could be inferred that the complete absence of the TnaA protein or the more subtle loss of its enzymatic function (i.e., indole production) is not sufficient or required to impose heat resistance, indicating that the evolved thaA variants should be considered subtle gain-of-function alleles.

In further consolidation of this causality and the need for thaA $A^{\Delta 106}$ to actually become expressed, we also observed that preventing tna $A^{\Delta 106}$ expression by depriving the growth medium of tryptophan (i.e., the inducer of the tnaCAB operon [21, 22]) abrogated the heat resistance effect (Fig. S1). Moreover, in tryptone soy broth (TSB) batch cultures, induction of the tnaCAB operon naturally occurs toward early stationary phase (when glucose becomes depleted and catabolite repression is alleviated [23-25]), which also corresponds in timing to the rise in PA-containing cells in the selected tnaA mutants (Fig. 2B). In contrast, induction of the tnaCAB operon already occurs in mid-exponential-phase LB in batch cultures (where amino acids are the main carbon source [26]), and exponential-phase heat resistance in the tnaA $A^{\Delta 106}$ mutant could accordingly be observed in LB but not in TSB medium (Fig. S2).

Compromised TnaA folding fidelity boosts the level of heat shock proteins. We subsequently hypothesized that the protein aggregation directly or indirectly inflicted by TnaA variants could lead to activation of the heat shock response, which in turn would provide the cell with increased heat resistance, while simultaneously leading to the emergence of PAs in the cell. Subsequent Western blot analysis indeed revealed that MG1655 $\Delta$ rpoS equipped with tna $A^{\Delta 106}$ displayed increased levels of heat shock proteins (HSPs) such as DnaK and GroEL compared to MG1655 $\Delta$ rpoS equipped with either tnaA ${ }^{W T}$ or tna $A^{K 270 A}$ (Fig. 4A). Moreover, the increase in DnaK and GroEL levels was 
A

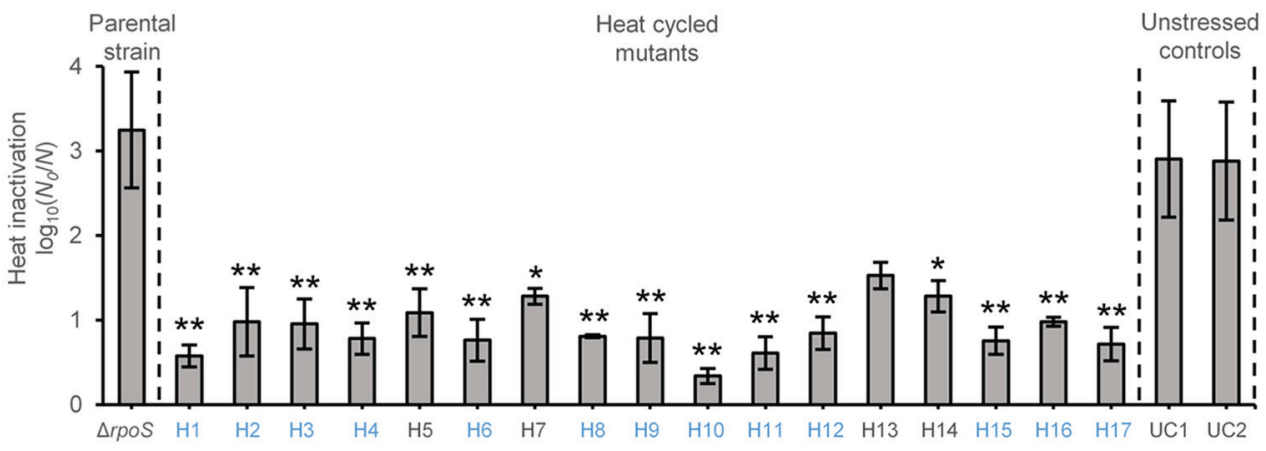

B
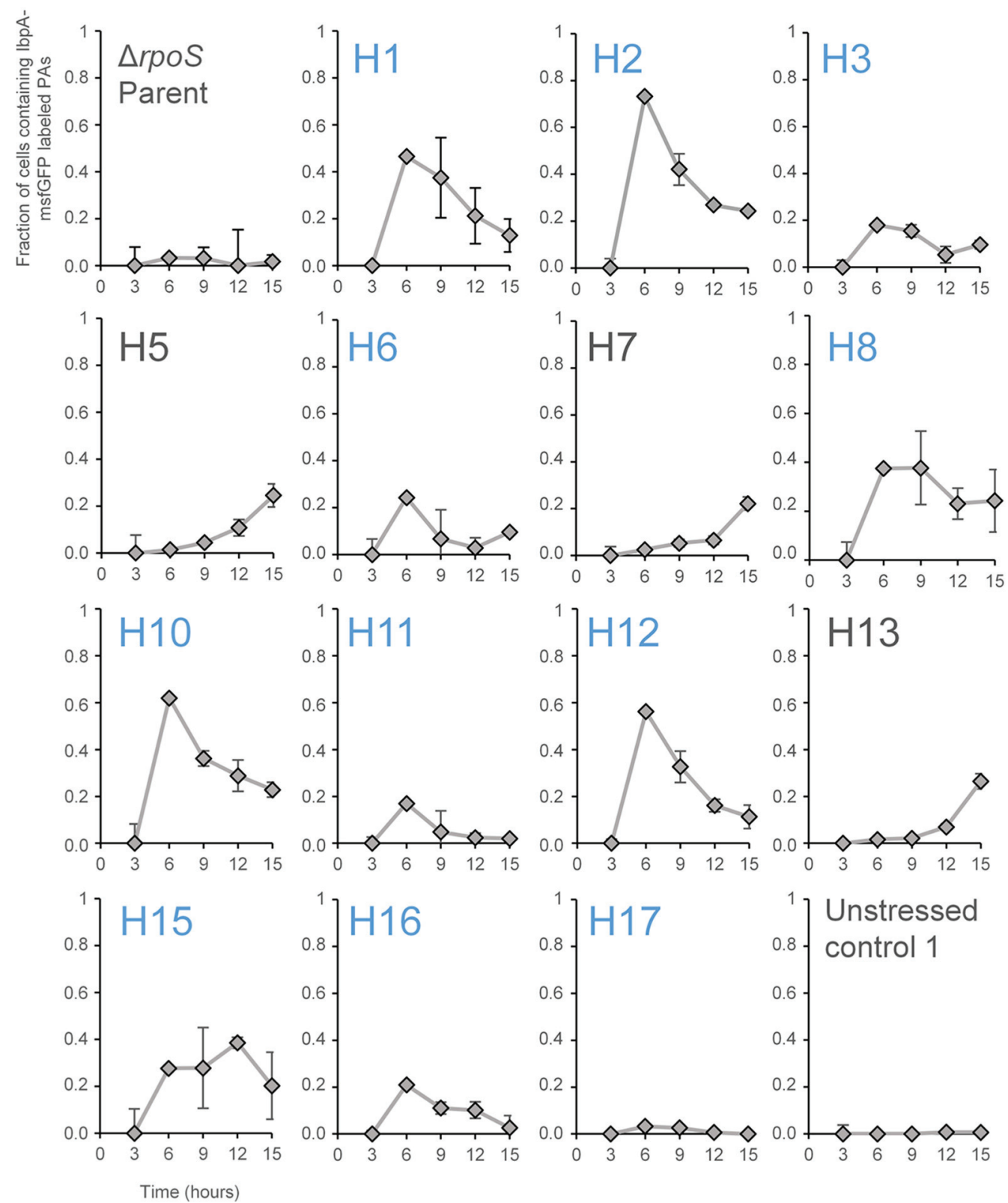
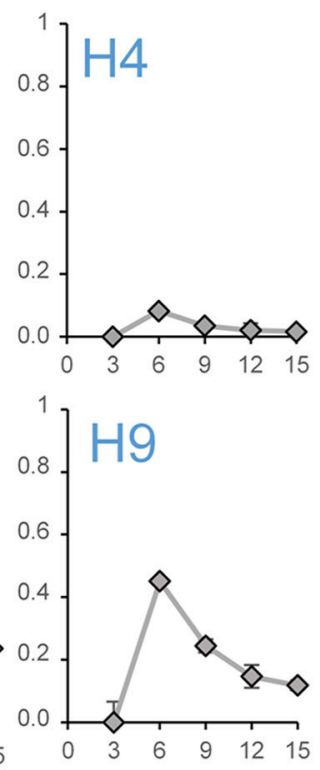

C
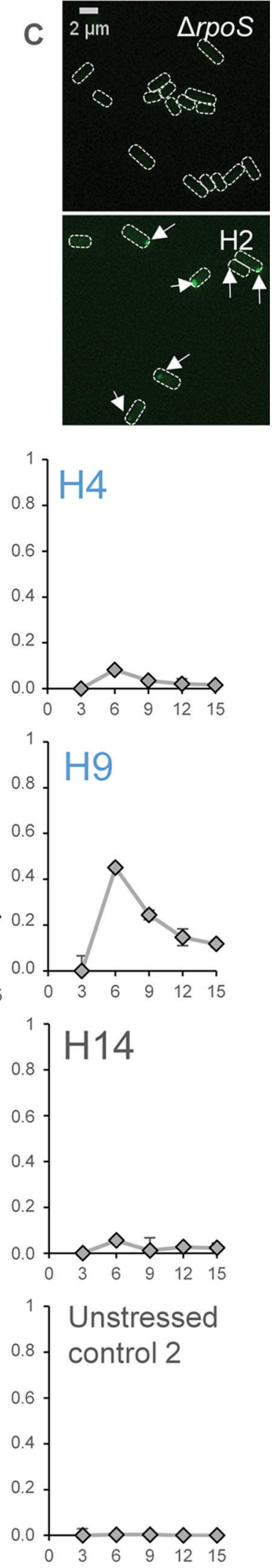

FIG 2 Mutants $\mathrm{H} 1$ to $\mathrm{H} 17$ were derived as single clones from independent lineages of the MG1655 $\Delta r p o S$ ibpA-msfGFP parental strain that were subjected to successive cycles of increasingly severe heat shocks (ranging from 15 min at $51^{\circ} \mathrm{C}$ to $55^{\circ} \mathrm{C}$ with $0.5^{\circ} \mathrm{C}$ increments) with intermittent 
similar to the included positive control of an MG1655 $\Delta r p o S r p o H^{154 T}$ mutant equipped with a constitutively active variant of the heat shock response sigma factor $\mathrm{RpoH}$ (Fig. 4A) (27). In fact, the extent of increased heat resistance of the $\Delta r p o S t n a A^{\Delta 106}$ mutant coincided with that of the $\Delta r p o S r p o H^{154 T}$ strain (Fig. 4B).

To more closely and causally link the expression of TnaA variants to induction of PAs, HSPs, and heat resistance, MG1655 (i.e., now bearing its wild type $\sigma^{\mathrm{s}}$ general stress response regulator) was chromosomally equipped with different reporter constructs and different tnaA alleles (or the $\mathrm{rpoH}^{154 T}$ allele as an HSP upregulated control), after which corresponding exponential-phase cultures were shortly induced with tryptophan and examined for (i) thaCAB promoter activity (using the $P_{\text {tnaCAB }}$-msfGFP reporter; Fig. 5A), (ii) heat resistance (Fig. 5B), (iii) HSP expression level (using the RpoHcontrolled $P_{h t p G}$-msfGFP reporter; Fig. 5C), and (iv) appearance of PAs (using the IbpAmsfGFP reporter; Fig. 5D). Next to tna $A^{W T}$, the examined thaA alleles included evolved allele tna $A^{\Delta 106}$ (causing a very high fraction of PA-bearing cells; Fig. $3 \mathrm{~B}$ ), evolved allele tna $A^{259 f 5}$ (causing a low fraction of PA-bearing cells and emerged in heat resistant mutant H17; Fig. 2B), or tnaA ${ }^{K 270 A}$ (expressing the catalytically compromised TnaA variant). While the short exposure to tryptophan clearly induced all TnaA variants (Fig. 5A), only induction of $\mathrm{TnaA}^{\Delta 106}$ and $\mathrm{TnaA}^{259 \mathrm{fs}}$ coincided with induction of heat resistance (Fig. 5B), HSPs (Fig. 5C), and PAs (Fig. 5D). In fact, the heat resistance and HSP induction of MG1655 tnaA $A^{\Delta 106}$ and MG1655 tnaA ${ }^{259 f s}$ mutants could rival those of MG1655 $r p o H^{154 T}$ in which HSPs and heat resistance are constitutively upregulated in the absence of any obvious protein aggregation (Fig. 5B to D). Additionally, these results also indicate that $\sigma^{\mathrm{s}}$ deficiency is not a requirement for these thaA alleles to confer their protective effect.

To independently validate these findings across more of the selected thaA alleles, both control alleles (i.e., tnaA $A^{W T}$ and $t n a A^{K 270 A}$ ) and a set of heat-selected alleles (i.e.,

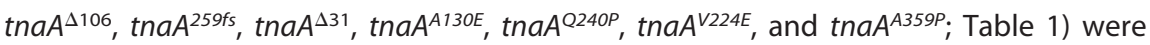
individually cloned downstream of the IPTG (isopropyl- $\beta$-D-thiogalactopyranoside)controlled $\mathrm{P}_{\text {trc }}$ promoter in the pTrc99A vector and transformed to the MG1655 $\mathrm{AlacY}$ ibpA-msfGFP $\mathrm{P}_{\text {dnak }}$-mScarlet-I (Fig. 6) and MG1655 $\Delta$ lacY dnaK-msfGFP (Fig. 7) reporter strains. Exponential-phase cultures of the resulting strains were subsequently induced with IPTG and examined for (i) heat resistance (Fig. 6A), (ii) HSP expression level (via the RpoH-controlled $P_{\text {dnak }}-m S$ carlet-I reporter in Fig. $6 \mathrm{~B}$ and the DnaK-msfGFP reporter in Fig. 7), (iii) appearance of PAs (via the IbpA-msfGFP reporter; Fig. 6C), and (iv) PA content (as approached by SDS-PAGE and mass spectrometry analysis of the insoluble and soluble protein content; Fig. $6 \mathrm{D}$ and $\mathrm{E}$ and Fig. S3). This confirmed that ectopic expression of the heat-selected tnaA alleles led to increased heat resistance (Fig. 6A) and increased levels of HSPs (Fig. 6B and Fig. 7) and PAs (Fig. 6C) compared to the tnaA ${ }^{W T}$ and $t n a A^{K 270 A}$ alleles. In two of the heat-selected thaA alleles (tnaA ${ }^{Q 240 P}$ and tna $\left.A^{V 224 E}\right)$, the increase in heat resistance was found not to be statistically significant (Fig. 6A), presumably because plasmid-based overexpression of $T$ naA $A^{Q 240 P}$ and TnaA ${ }^{\mathrm{V} 224 \mathrm{E}}$ coincided with very large amounts of misfolded proteins and PAs (Fig. 6C) that likely imposed a burden on the cell. These experiments also indicate the domi-

FIG 2 Legend (Continued)

outgrowth to stationary phase in TSB. As a control, UC1 and UC2 were derived as single clones from independent lineages of MG1655 $\Delta r p o S$ ibpAmsfGFP that were similarly cycled but without being exposed to heat stress. (A) Logarithmic reduction factor after exposure to heat $\left(55^{\circ} \mathrm{C}\right.$ for 15 min) of stationary-phase TSB cultures of the parental MG1655 $\Delta$ rpoS ibpA-msfGFP strain and its indicated derivatives. Asterisks indicate a statistically significant decrease in the logarithmic reduction factor compared to the parental strain (Tukey HSD post hoc test; $\left.{ }^{*}, P \leq 0.05 ;{ }^{* *}, P \leq 0.01\right)$. (B) The fraction of cells containing a fluorescent IbpA-msfGFP labeled PA in the parental MG1655 $\Delta$ rpoS ibpA-msfGFP strain and its indicated derivatives was determined microscopically by sampling a growing TSB culture every $3 \mathrm{~h}$ after reinoculating $1 / 100$ from an overnight culture. Out of the 17 heatselected mutants, $14(\mathrm{H} 1, \mathrm{H} 2, \mathrm{H} 3, \mathrm{H} 5, \mathrm{H} 6, \mathrm{H} 7, \mathrm{H} 8, \mathrm{H} 9, \mathrm{H} 10, \mathrm{H} 11, \mathrm{H} 12, \mathrm{H} 13, \mathrm{H} 15$, and $\mathrm{H} 16)$ could be considered as having aberrant cellular PA management compared to the controls on the basis of the IbpA-msfGFP reporter. On average, 100.96 cells were observed per strain and time point per independent experiment, and all sample sizes were between 64 and 148 cells. For panels $A$ and $B$, the displayed means were determined over three independent experiments, and the error bars indicate the standard error over these experiments. Blue labels indicate mutants with mutations in their tnaA allele as determined by whole-genome sequencing. (C) Representative GFP epifluorescence (reporting IbpA-msfGFP expression and localization) images of MG1655 $\triangle$ rpoS ibpA-msfGFP and one of its heat-selected mutants ( $\mathrm{H} 2$ ) after $6 \mathrm{~h}$ of exponential growth in TSB. Cell outlines are shown in white, and the white arrows indicate IbpA-msfGFP labeled PAs. Scale bar corresponds to $2 \mu \mathrm{m}$. 


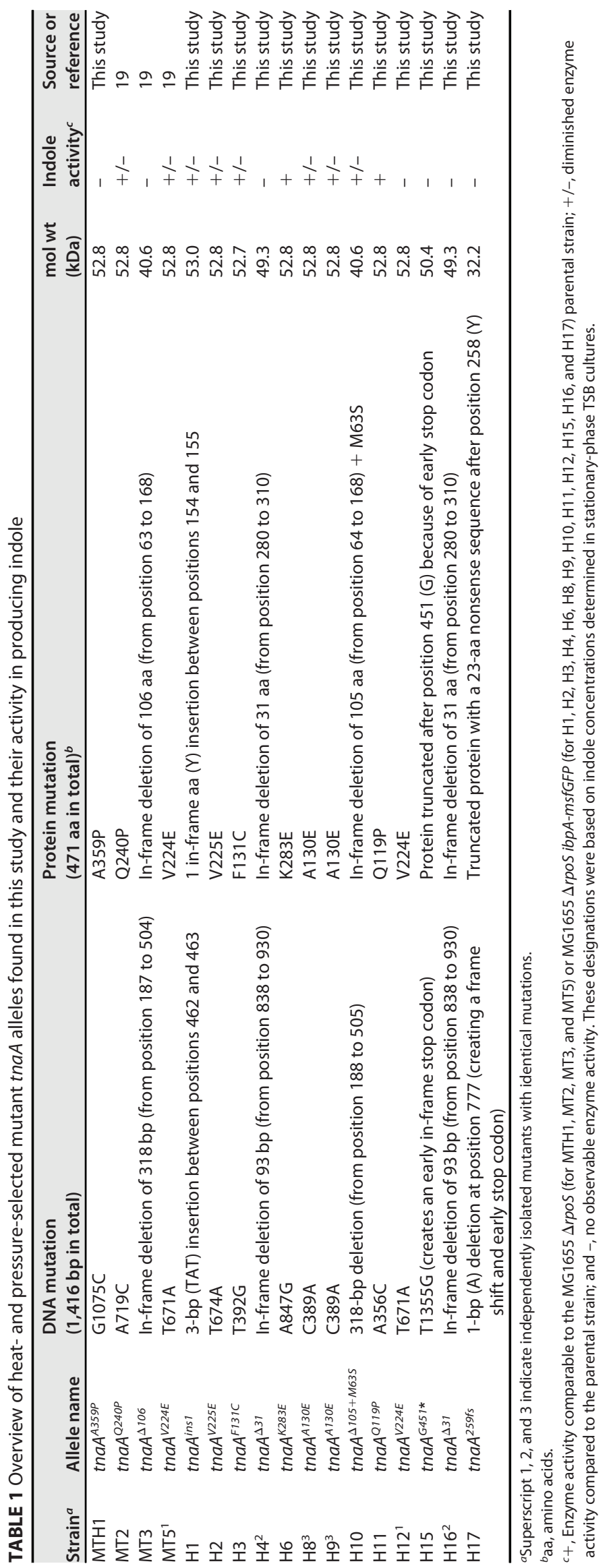




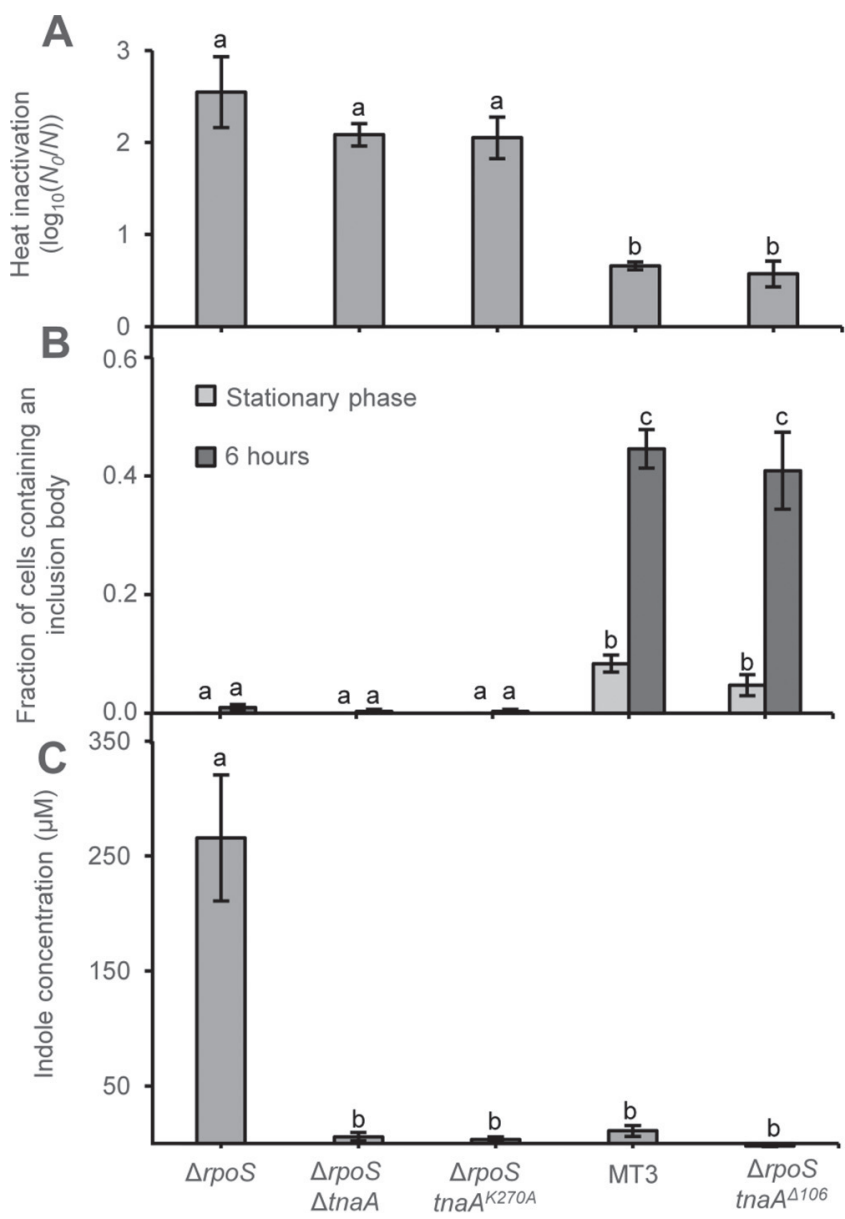

FIG 3 (A) The logarithmic reduction factor after exposure to heat $\left(55^{\circ} \mathrm{C}\right.$ for $\left.15 \mathrm{~min}\right)$ of stationaryphase TSB cultures of MG1655 $\Delta r p o S$, MG1655 $\Delta r p o S \Delta$ tnaA, MG1655 $\Delta r p o S$ tnaA ${ }^{K 270 A}$ (harboring a catalytically inactive TnaA variant), the originally evolved MT3 mutant (harboring the tnaA ${ }^{\Delta 106}$ allele), and the reconstructed MG1655 $\Delta r p o S$ tnaA $A^{\Delta 106}$ strain. (B) The fraction of cells that contain an inclusion body visible in phase contrast was determined for the indicated strains after growth in TSB to stationary phase and $6 \mathrm{~h}$ after reinoculating $1 / 100$ in fresh TSB medium. On average, 95.9 cells were observed per strain and condition per independent experiment, and all sample sizes were between 80 and 110 cells. (C) Indole concentrations produced by the indicated strains after growth in TSB to stationary phase. For panels $A$ to $C$, the displayed means were determined over three independent experiments, and the error bars indicate the standard error over these experiments. Within each panel, different letters indicate statistically significant (Tukey HSD post hoc test, $P$ value $\leq 0.05$ ) differences among different strains and time points.

nance of the heat-selected thaA alleles over the chromosomal tnaA ${ }^{W T}$ allele that is still present in the reporter strains, which is in line with our hypothesis that these are gainof-function alleles.

Importantly, although the wild-type TnaA protein is known to be a soluble cytoplasmic protein, SDS-PAGE analysis of the insoluble and soluble protein fractions of the above-mentioned plasmid strains clearly revealed that the heat-selected TnaA ${ }^{\Delta 106}$ $\mathrm{TnaA}^{259 f 5}$, TnaA ${ }^{\Delta 31}$, TnaA ${ }^{\mathrm{A130E}}$, TnaA ${ }^{\mathrm{Q} 240 \mathrm{P}}$, TnaA ${ }^{\mathrm{V224E}}$, and TnaA ${ }^{\mathrm{A3} 59 \mathrm{P}}$ variants (in contrast to TnaA ${ }^{W T}$ and $T n A^{K 270 A}$ ) massively ended up in the insoluble protein fraction when their expression was induced with IPTG (Fig. 6D and E), indicating that these variants are themselves aggregation-prone and can thus serve as the direct molecular cause of HSP induction. The abundant presence of TnaA in the insoluble protein fraction was indeed confirmed by mass spectrometry analysis for the strains expressing TnaA ${ }^{\Delta 106}$ and TnaA ${ }^{25995}$. TnaA has been identified in the gel bands with a $100 \%$ protein probability (Fig. S3, Data Set S1). 

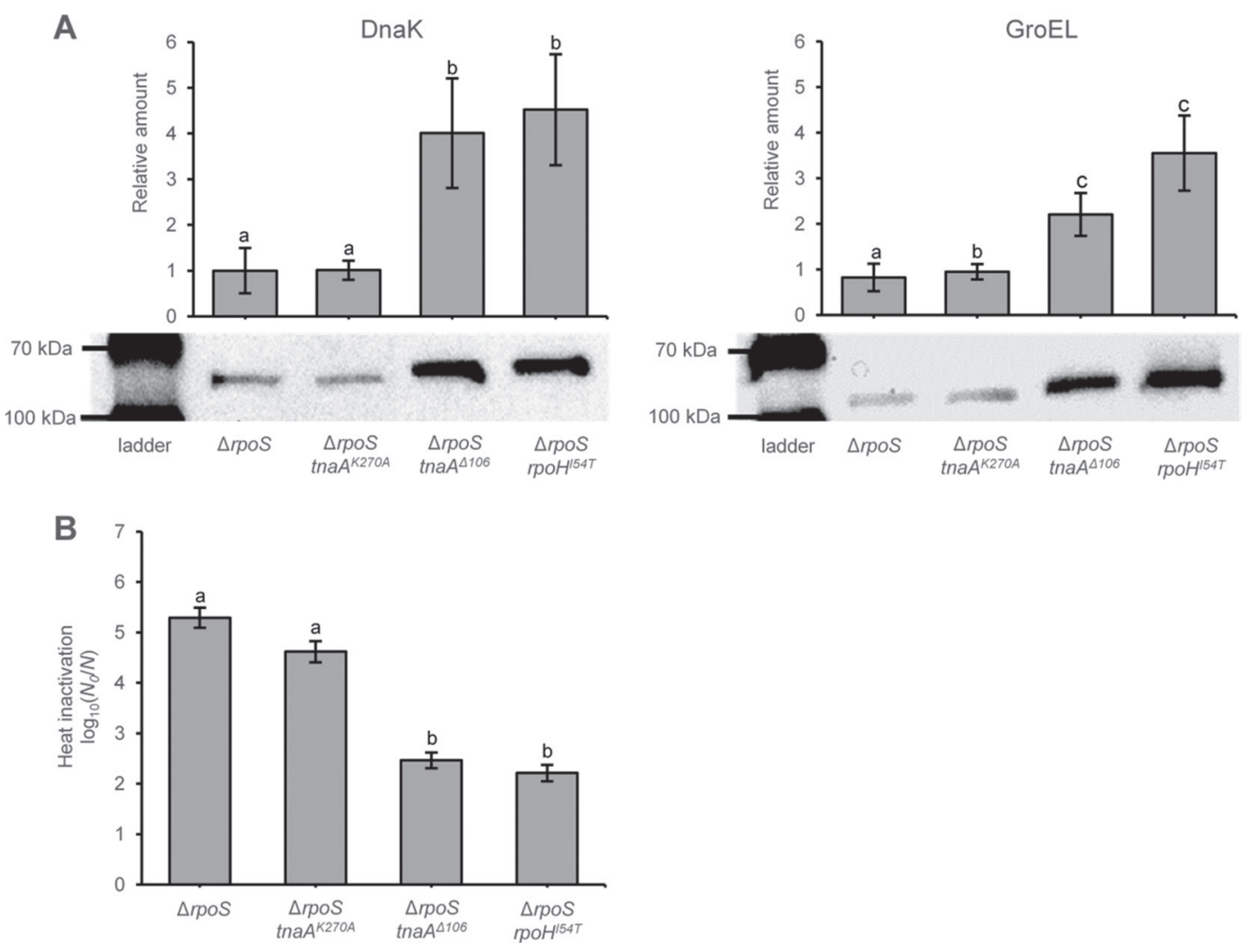

FIG 4 (A) Western blot analysis of DnaK and GroEL expression in stationary-phase cell lysates of MG1655 $\Delta r p o S$, MG1655 $\Delta r p o S$ tnaA ${ }^{K 270 A}$, MG1655 $\Delta r p o S$ tnaA $A^{\Delta 106}$, and MG1655 $\Delta r p o S r p o H^{154 T}$ grown in TSB. The data display the means of relative amounts of protein (as determined by the density of the bands after background subtraction) compared to the $\Delta r p o S$ parent determined over four independent experiments, and the error bars indicate the standard error over these experiments. Images below the graphs correspond to a representative gel from one of the independent experiments. (B) Logarithmic reduction factor after heat exposure $\left(56^{\circ} \mathrm{C}\right.$ for $\left.15 \mathrm{~min}\right)$ of the indicated strains grown to stationary phase in TSB. The displayed means were determined over three independent experiments, and the error bars indicate the standard error over these experiments. Different letters indicate statistically significant differences (Student's $t$ tests followed by Bonferroni correction, $P$ value $\leq 0.05)$ in the relative amount of protein $(A)$ or inactivation $(B)$ among strains.

In silico analysis of heat-selected TnaA variants. Closer examination of the heatselected full-length TnaA variants indicated that they typically incurred single amino acid substitutions to Glu or Pro (Table 2 and represented in Fig. S4), of which the residues (upon misplacement) were described to be particularly detrimental to overall protein structure (28). In fact, thermodynamic stability calculations (using FoldX [29]) revealed that each of the selected substitutions significantly destabilizes the native structure of the TnaA protein, while the catalytically compromised TnaA ${ }^{\text {K270A }}$ variant experiences no significant structural destabilization ( $\Delta \Delta \mathrm{G}$ scores in Table 2). Moreover, comparing the distribution of the FoldX values of the heat-selected mutants with the distributions obtained either from (i) all TnaA single substitutions that can theoretically be genetically accessed through a single point mutation or (ii) naturally occurring TnaA orthologs strongly suggests heat selection toward TnaA variants with severe structural destabilization (Fig. 8). In addition, the heat-selected substitutions had a zero or extremely low frequency of occurrence in the natural (and likely functional) orthologs (Table 2), further suggesting clear selective pressure toward structural disruption. As such, these findings further underscore that the heat-selected TnaA variants are indeed affected in their folding fidelity and inclined to trigger HSP expression because of this feature.

TnaA-PAs also provide epigenetically inheritable longer-term heat resistance. Time-lapse fluorescence microscopy monitoring of MG1655 cells chromosomally equipped with thaA $A^{\Delta 106}$ or tha $A^{259 f s}$ (and different reporter constructs) after halting 

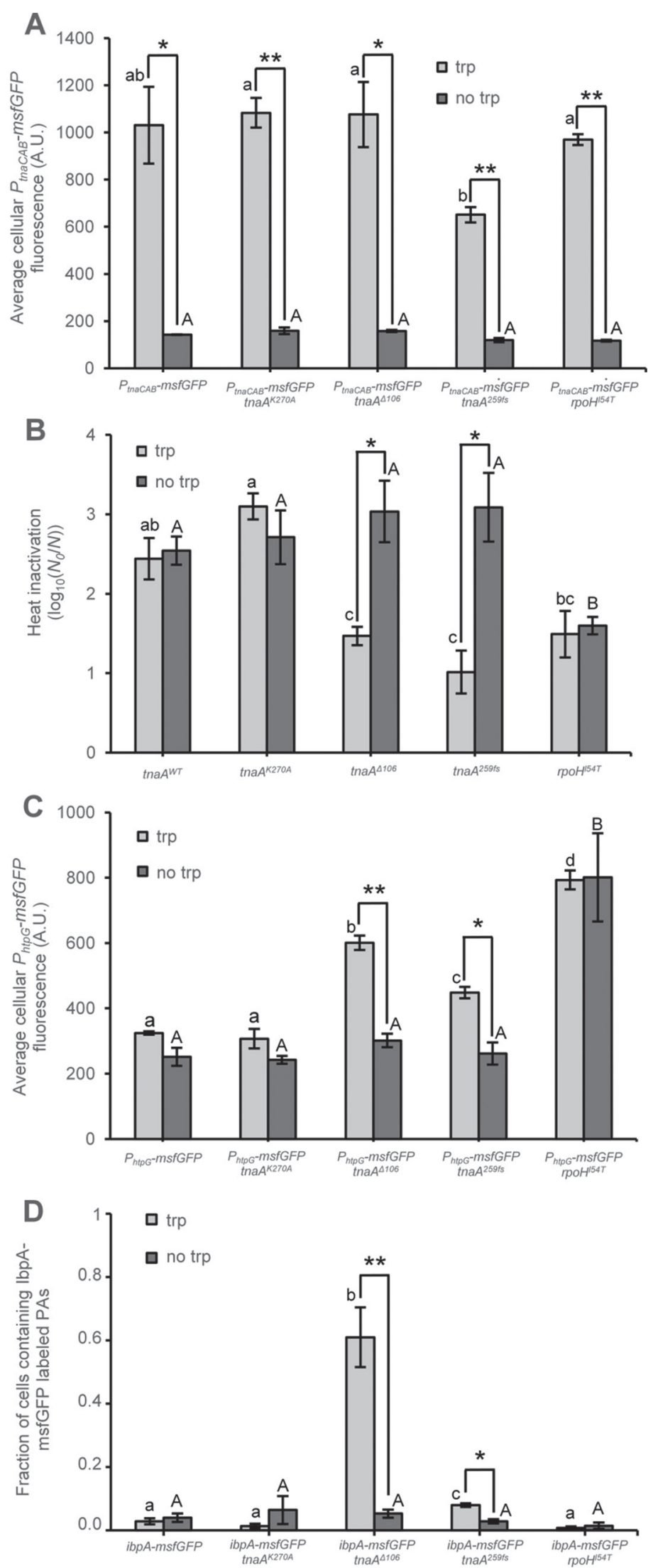

FIG 5 (A) Average cellular fluorescence of AB-grown exponential-phase populations of the MG1655 $P_{\text {tnaCAB}}-m s f G F P$ fluorescent reporter (harboring $t n a A^{W T}$ and $r p o H^{W T}$ ) and its $\operatorname{tna} A^{K 270 A}$, tnaA $A^{\Delta 106}$, tha $A^{259 f s}$,

(Continued on next page) 
tryptophan-mediated induction revealed that over subsequent generations HSP expression (as judged by the $P_{h t p G}$-msfGFP reporter) homogeneously returned to basal levels (shown for tnaA ${ }^{\Delta 106}$ in Fig. S5A). On the other hand, the formed TnaA-PAs (as judged by the IbpA-msfGFP reporter) segregated asymmetrically among sister cells, thereby creating PA-bearing $\left(\mathrm{PA}^{+}\right)$and PA-lacking $\left(\mathrm{PA}^{-}\right)$siblings (shown for tha ${ }^{\Delta 106}$ in Fig. S5B). The homogeneous extinction of HSP expression in an emerging microcolony (despite heterogeneous segregation of PAs) underscores that increased HSP expression is triggered by the initial production of misfolded TnaA variants, and not per se by the PA structures into which they assemble. Moreover, the heat resistance of MG1655 tna $A^{\Delta 106}$ and MG1655 tnaA ${ }^{259 f s}$ was similar (Fig. 5B), despite the fact that in MG1655 tna $A^{\Delta 106}$ the TnaA-PAs were more abundant (Fig. 5D), further emphasizing that the size or stability of the PA structures is not necessarily instructive for the level of HSP and heat resistance raised.

Nevertheless, we recently documented the occurrence of a different PA-based heat resistance phenomenon (18). In fact, asymmetric inheritance of an ancestral PA (i.e., stemming from a prior heat shock or prior expression of an aggregation-prone protein many generations before) was shown to epigenetically endow the $\mathrm{PA}^{+}$cell with improved heat resistance compared to its $\mathrm{PA}^{-}$siblings (18). In order to examine whether ancestral TnaAPAs could have such a similar protective effect, well after increased HSP expression coinciding with their original emergence has dampened out, MG1655 tnaA $A^{106}$ was tryptophan-induced for $2 \mathrm{~h}$ in a liquid culture and subsequently grown for $2 \mathrm{~h}$ on agarose pads lacking tryptophan. The resulting microcolonies, now typically consisting of one $\mathrm{PA}^{+}$and several $\mathrm{PA}^{-}$isogenic siblings (Fig. 9A), were subsequently heat challenged, after which, survival and resuscitation time of both types of cells were compared. This clearly revealed (i) that $\mathrm{PA}^{+}$siblings were endowed with an (on average) 1.9-fold increased survival chance (Fig. 9B), while (ii) the $\mathrm{PA}^{+}$survivors were furthermore endowed with an (on average) $2.5 \mathrm{~h}$ decrease in resuscitation time compared to $\mathrm{PA}^{-}$siblings (Fig. $9 \mathrm{C}$ ). In fact, survival frequency and resuscitation time of $\mathrm{PA}^{-}$siblings were similar to those of MG1655 tna $A^{\Delta 106}$ cells not previously triggered with tryptophan (Fig. 9B and C), indicating that the contribution of the ancestrally elevated HSP levels in the tryptophan-triggered $\mathrm{PA}^{-}$cells meanwhile indeed dampened out completely.

As such, the aggregating TnaA variants cause (i) an immediate population-level heat resistance resulting from the surge in HSP levels and (ii) a secondary subpopulation-level heat resistance in those siblings retaining/inheriting the ancestral TnaA-PA many generations afterward.

FIG 5 Legend (Continued)

or $r p o H^{154 T}$ derivatives with (trp; light gray) or without (no trp; dark gray) tryptophan induction. The average number of observed cells per strain and condition per independent experiment was 347.0, and sample sizes were always between 143 and 745 cells. (B) Logarithmic reduction factor after exposure to heat $\left(54.5^{\circ} \mathrm{C}\right.$ for $15 \mathrm{~min}$, recovery on $\mathrm{LB}$ agar) of $\mathrm{AB}$-grown exponential-phase populations of MG1655 (harboring tna $A^{W T}$ and $r p o H^{W T}$ ) and its $\operatorname{tnaA}^{K 270 A}$, thaA $A^{\Delta 106}$, thaA ${ }^{259 f s}$, or rpoH $H^{154 T}$ derivatives with (trp; light gray) or without (no trp; dark gray) tryptophan induction. (C) Average cellular fluorescence of AB-grown exponential-phase populations of the MG1655 $P_{h t p G}-m s f G F P$ fluorescent reporter (harboring tna $A^{W T}$ and $r p o H^{W T}$ ) and its tna $A^{K 270 A}$, tha $A^{\Delta 106}$, tna $A^{259 f s}$, or rpoH $H^{154 T}$ derivatives with (trp; light gray) or without (no trp; dark gray) tryptophan induction. The average number of observed cells per strain and condition per independent experiment was 421.7, and sample sizes were always between 116 and 965 cells. (D) Fraction of cells containing an IbpA-msfGFP labeled PA of AB-grown exponential-phase populations of the MG1655 ibpA-msfGFP fluorescent reporter (harboring tna $A^{W T}$ and $r p o H^{W T}$ ) and its tna $A^{K 270 A}$, tna $A^{\Delta 106}$, tha $A^{259 f s}$, or rpoH ${ }^{154 T}$ derivatives with (trp; light gray) or without (no trp; dark gray) tryptophan induction. On average, 91.3 cells were observed per strain and condition per independent experiment, and all sample sizes were between 50 and 106 cells. For all panels, strains were grown to exponential phase in $A B$ medium (i.e., $4 \mathrm{~h}$ of growth after $1 / 1,000$ dilution of stationary-phase cultures) with or without addition of $1.25 \mathrm{mM}$ tryptophan for the last $2 \mathrm{~h}$ of growth. For all panels, the displayed means were determined over three independent experiments, and the error bars indicate the standard error over these experiments. Asterisks indicate a statistically significant difference for induced compared to noninduced populations of the same strain (Student's $t$ tests followed by Bonferroni correction; ${ }^{*}, P \leq 0.05 ;{ }^{*}, P \leq 0.01$ ), while different lowercase (for tryptophan-induced populations) or capital (for noninduced populations) letters indicate statistically significant differences among strains (Student's $t$ tests followed by Bonferroni correction, $P$ value $\leq 0.05$ ). 


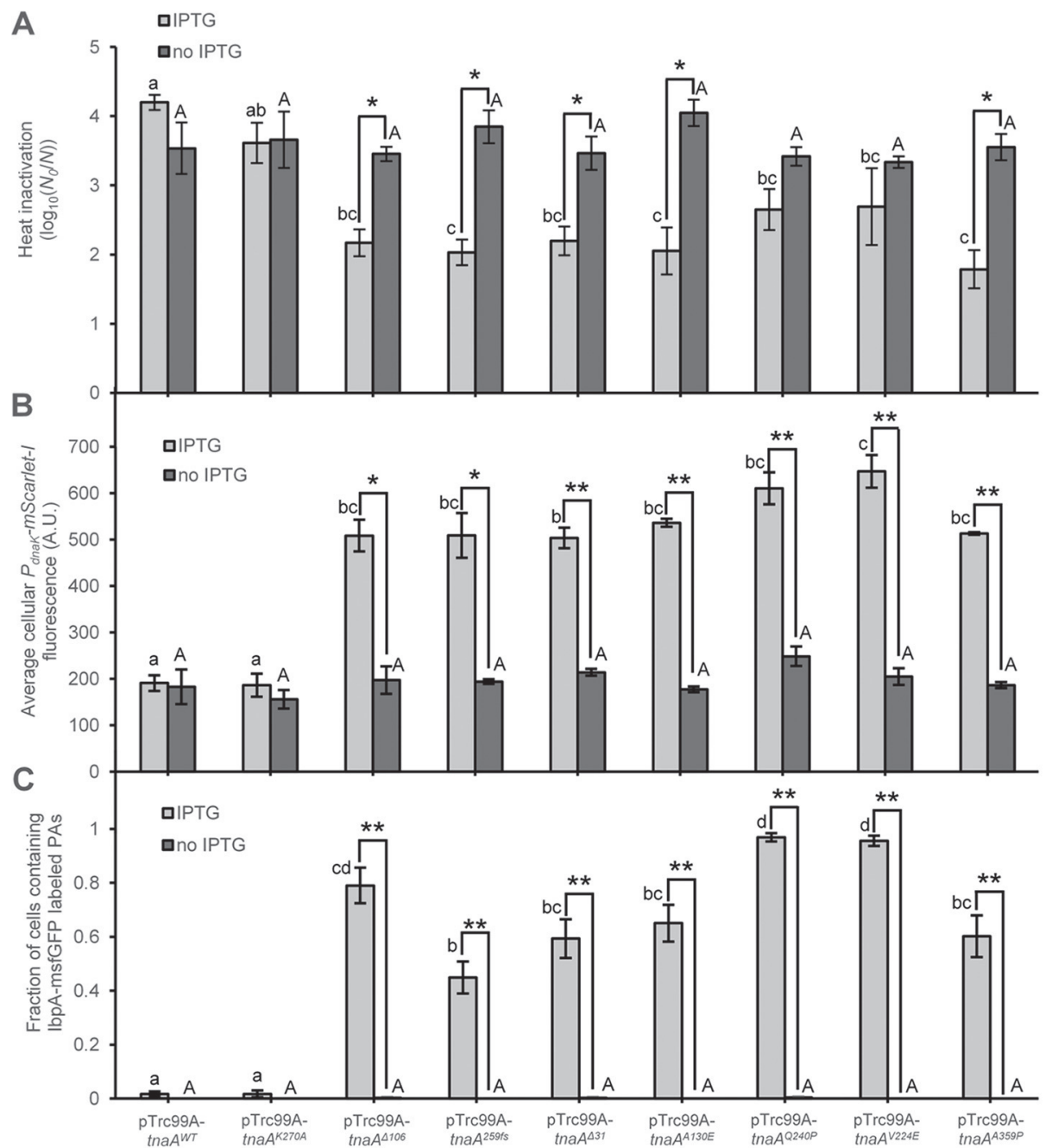

D Insoluble fraction

E Soluble fraction

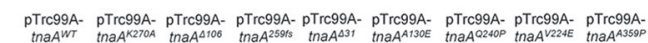

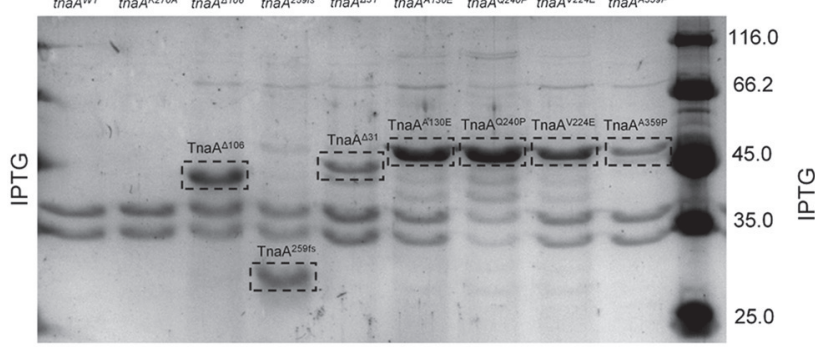

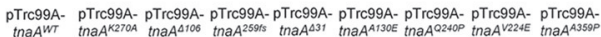

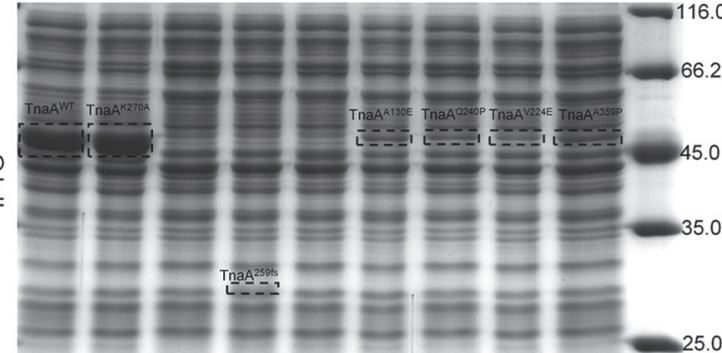

pTrc99A- pTrc99A- pTrc99A- pTrc99A- pTrc99A- pTrc99A- PTrc99A- pTrc99A- pTrc99A-

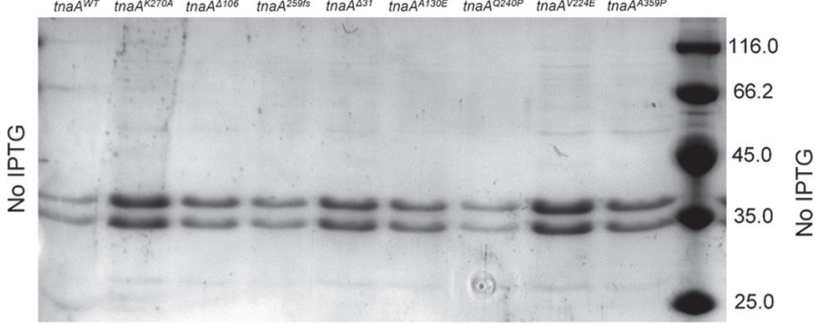

pTrc99A- pTrc99A- pTrc99A- pTrc99A- pTrc99A- pTrc99A- pTrc99A- pTrc99A- PTrc99A-
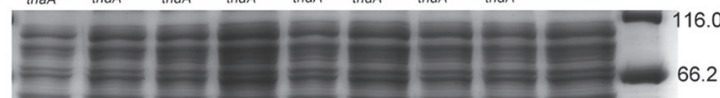

()

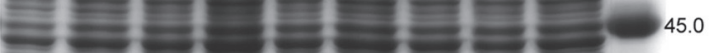

$=$

FIG 6 (A) Logarithmic reduction factor after exposure to heat $\left(55^{\circ} \mathrm{C}\right.$ for $15 \mathrm{~min}$, recovery on TSA) of AB-grown exponential-phase populations of MG1655 $\Delta$ lacY ibpA-msfGFP $\mathrm{P}_{\text {dnak }}$-mScarlet-I carrying the indicated pTrc99A-tnaA plasmids with (IPTG; light gray) or 


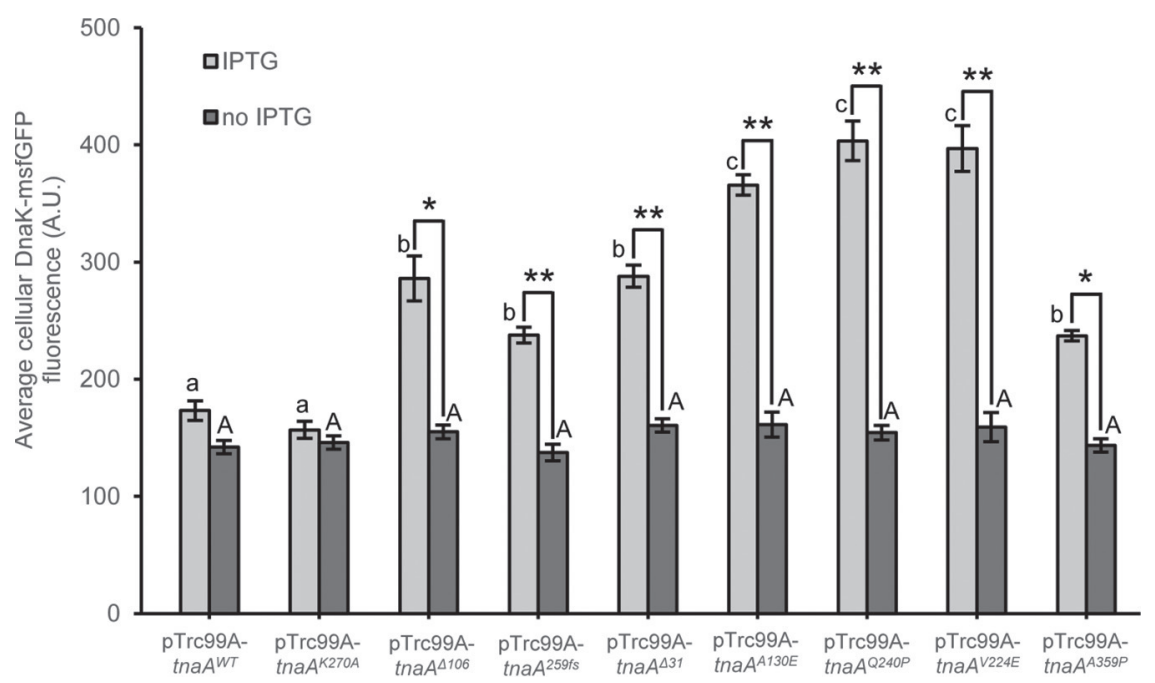

FIG 7 Average cellular msfGFP fluorescence of AB-grown exponential-phase populations of MG1655 $\Delta$ lacY dnaK-msfGFP carrying the indicated pTrc99A-tnaA plasmids with (IPTG; light gray) or without (no IPTG; dark gray) IPTG induction. The average number of observed cells per strain and condition per independent experiment was 296.6, and sample sizes were always between 47 and 1,032 cells. Strains were grown to exponential phase in $A B$ medium (i.e., $4.5 \mathrm{~h}$ of growth after 1/1,000 dilution of stationary-phase cultures) with or without addition of $100 \mu \mathrm{M}$ IPTG for the last $2 \mathrm{~h}$ of growth. The displayed means were determined over three independent experiments, and the error bars indicate the standard error over these experiments. Asterisks indicate a statistically significant difference for induced compared to noninduced populations of the same strain (Student's $t$ tests followed by Bonferroni correction; ${ }^{*}, P \leq 0.05 ;{ }^{* *}, P \leq 0.01$ ), while different lowercase (for IPTG-induced populations) or capital (for noninduced populations) letters indicate statistically significant differences among strains (Tukey HSD post hoc test, $P$ value $\leq 0.05$ ).

\section{DISCUSSION}

Our results essentially indicate that adaptive evolution can capitalize on erosive mutations that alter protein folding fidelity and subsequent aggregation dynamics, thereby enabling cells to autonomously raise HSP expression and boost their robustness against future proteotoxic stresses. This (bottom-up) adaptive evolutionary strategy deviates from common expectations to find (top-down) upregulation of the heat shock response through mutations in its master regulators (such as the dedicated $\mathrm{RpoH}$ sigma factor), but instead relies on preemptive self-inflicted damage. Indeed, while the protein quality control network is known to alleviate the impact of a proteotoxic stress by restoring the stability of stress-affected proteins, our findings now (reciprocally) indicate that the acquisition of mutations sacrificing protein folding fidel-

FIG 6 Legend (Continued)

without (no IPTG; dark gray) IPTG induction. (B) Average cellular mScarlet-I fluorescence of AB-grown exponential-phase populations of MG1655 $\Delta$ lacY ibpA-msfGFP $P_{\text {dnak }}$-mScarlet-l carrying the indicated pTrc99A-tnaA plasmids with (IPTG; light gray) or without (no IPTG; dark gray) IPTG induction. The average number of observed cells per strain and condition per independent experiment was 427.3, and sample sizes were always between 59 and 1193 cells. (C) Fraction of cells containing an IbpA-msfGFP labeled PA of AB-grown exponential-phase populations of MG1655 $\Delta$ lacY ibpA-msfGFP $\mathrm{P}_{\text {dnak }}$-mScarlet-l carrying the indicated pTrc99A-tnaA plasmids with (IPTG; light gray) or without (no IPTG; dark gray) IPTG induction. On average, 95.4 cells were observed per strain and condition per independent experiment, and all sample sizes were between 90 and 108 cells. (D) Representative SDS-PAGE analysis of insoluble protein fractions of AB-grown exponential-phase populations of MG1655 SlacY dnaK-msfGFP carrying the indicated pTrc99A-tnaA plasmids with (top) and without (bottom) IPTG induction. (E) Representative SDS-PAGE analysis of soluble protein fractions of AB-grown exponential-phase populations of MG1655 $\Delta / a c Y$ dnaK-msfGFP carrying the indicated pTrc99A-tnaA plasmids with (top) and without (bottom) IPTG induction. For panels D and E, numbers in black indicate the molecular weights of the protein ladder bands (in $\mathrm{kDa}$ ) and the dashed rectangles indicate the bands presumed to correspond to the indicated TnaA variants (for molecular weights, see Table 1). For all panels, strains were grown to exponential phase in $A B$ medium (i.e., $4.5 \mathrm{~h}$ of growth after 1/1,000 dilution of stationary-phase cultures) with or without addition of $100 \mu \mathrm{M}$ IPTG for the last $2 \mathrm{~h}$ of growth. For panels $\mathrm{A}$ to $\mathrm{C}$, the displayed means were determined over three independent experiments, and the error bars indicate the standard error over these experiments. Asterisks indicate a statistically significant difference for induced compared to noninduced populations of the same strain (Student's $t$ tests followed by Bonferroni correction; ${ }^{*}, P \leq 0.05$; ${ }^{*}, P \leq 0.01$ ), while different lowercase (for IPTG-induced populations) or capital (for noninduced populations) letters indicate statistically significant differences among strains (Tukey HSD post hoc test, $P$ value $\leq 0.05$ ). 
TABLE 2 Characterization of heat-selected TnaA variants harboring a single amino acid substitution $^{a}$

\begin{tabular}{|c|c|c|c|c|}
\hline Mutation & $\% \mathrm{MT}^{b}$ & $\% \mathrm{WT}^{\mathrm{c}}$ & Occurring AAs in orthologous cluster ${ }^{d}$ & FoldX $\Delta \Delta \mathrm{G}(\mathrm{kcal} / \mathrm{mol})$ \\
\hline Q119P & 0.0 & 36.2 & $\mathrm{~A}, \mathrm{G}, \mathrm{L}, \mathrm{V}, \mathrm{I}, \mathrm{C}, \mathrm{M}, \mathrm{Y}, \mathrm{D}, \mathrm{E}, \mathrm{R}, \mathrm{K}, \mathrm{Q}, \mathrm{N}, \mathrm{H}$ & 3.19 \\
\hline A130E & 0.0 & 32.0 & $A, L, V, I, S, T, C, F$ & 9.83 \\
\hline F131C & 0.0 & 28.0 & $A, L, V, I, F, P$ & 4.41 \\
\hline V224E & 0.0 & 50.0 & $L, V, I, M, F, Y$ & 6.91 \\
\hline V225E & 0.0 & 46.8 & $L, V, I, C, M, W, F, Y, D, Q, N$ & 5.47 \\
\hline Q240P & 0.0 & 54.6 & $A, L, V, I, S, T, M, Y, E, R, K, Q, N, H$ & 5.20 \\
\hline K283E & 0.5 & 47.2 & $A, S, T, D, E, R, K, N, H$ & 2.65 \\
\hline A359P & 0.0 & 89.4 & $A, G$ & 4.67 \\
\hline $\mathrm{K} 270 \mathrm{~A}$ & 0.0 & 100.0 & & 0.01 \\
\hline
\end{tabular}

aThe catalytically compromised TnaA ${ }^{\mathrm{K} 270 \mathrm{~A}}$ variant is included in the bottom row for comparison.

bPercentage of entries in the alignment where the mutant residue occurs.

cPercentage of entries where the wild-type residue occurs.

aa, amino acids.

ity of even a single nonessential protein presents an evolutionary mechanism to tune the basal levels and readiness of the chaperone network. More generally, our findings indicate that some loss-of-function mutations might as well be orthogonal gain-offunction mutations, further implying that eroding genes with a degenerating sequence (e.g., pseudogenes that are no longer under stringent selection [30]) are not necessarily on route to being evolutionarily purged and lost from the genome but can actually be actively retained by natural selection.

Interestingly, the reason for the tnaA allele being an apparent hot spot for such folding-compromising mutations in our setup is not related to the enzymatic activity of the encoded protein (Fig. $3 \mathrm{C}$ and Table 1), despite the fact that indole is otherwise known as a signal molecule with a myriad of physiological effects (e.g., on plasmid stability [31], cell division [32], antibiotic tolerance [33, 34], virulence [35] and biofilm formation [36]). In fact, it rather, seems related to the fact that thaA presents a nonessential (i.e., disposable) gene that becomes fully expressed toward the early stationary phase of TSB-grown cells (i.e., when alleviated catabolite repression and the presence of tryptophan boost the thaCAB operon [23-25]). Since, during our iterative directed

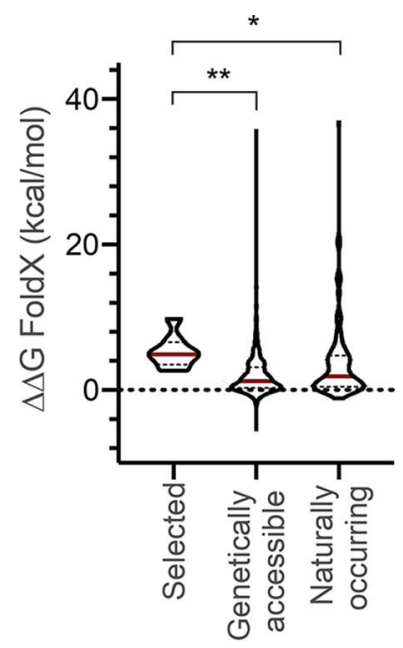

FIG 8 Violin plots of the predicted effects on TnaA stability as calculated through FoldX, using the TnaA pbd-structure 2oqx (57). The stability of heat-selected TnaA variants with single amino acid substitutions is compared to (i) all possible variants that are genetically accessible through a single point mutation and (ii) variants that are found to be naturally occurring in TnaA orthologues. The dashed horizontal line indicates a $\Delta \Delta \mathrm{G}$ value of 0 (corresponding to variants with unaltered stability compared to TnaA ${ }^{\mathrm{WT}}$ ). Within each violin plot, median values are indicated by a red horizontal line, while dashed horizontal lines indicate 1st and 3rd quartiles. Significance was determined with a Kruskal-Wallis test with post hoc Dunn's multiple-comparison test $\left({ }^{*}, P \leq 0.05 ;{ }^{* *}, P \leq 0.01\right)$. 
A

Phase contrast

IbpA-msfGFP

Overlay
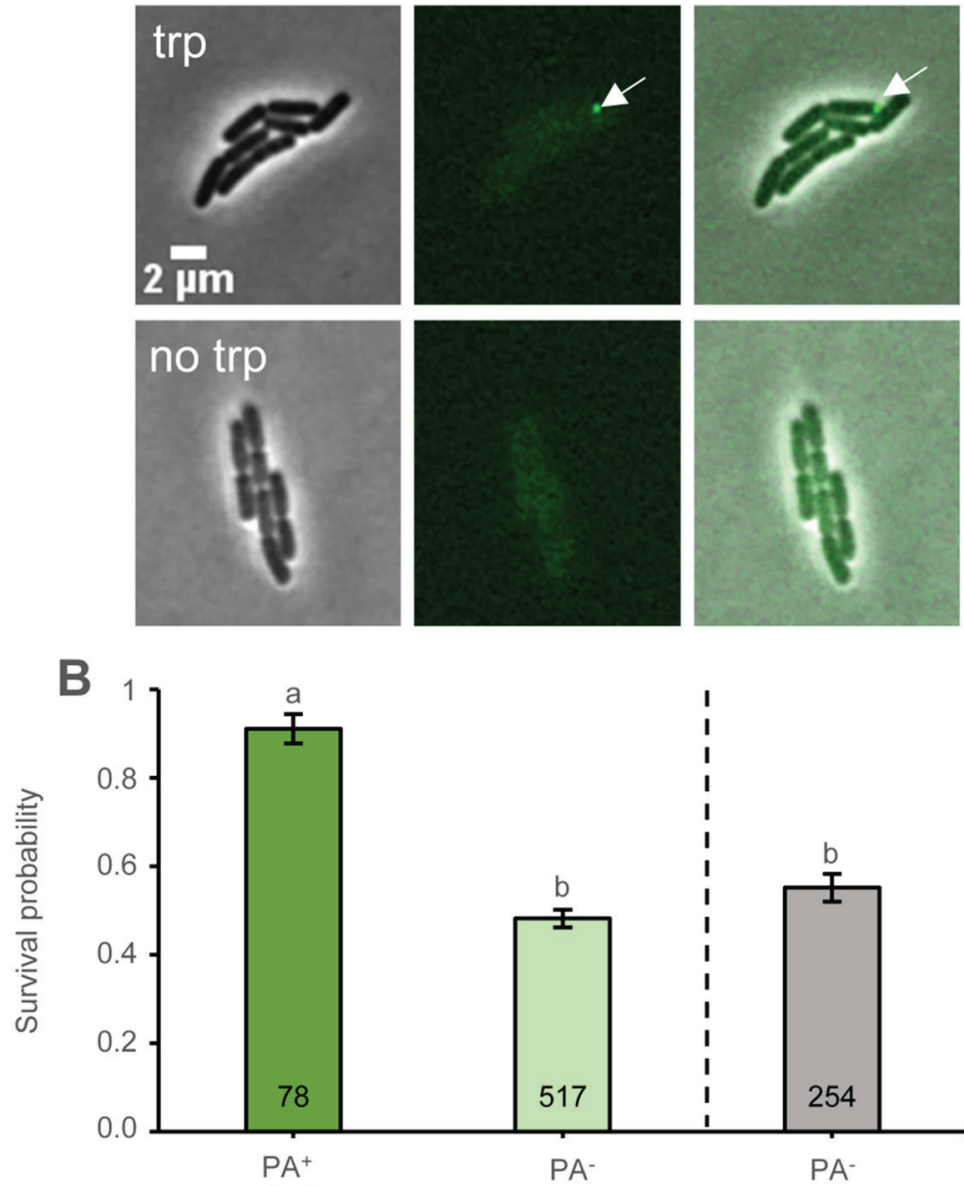

(trp)

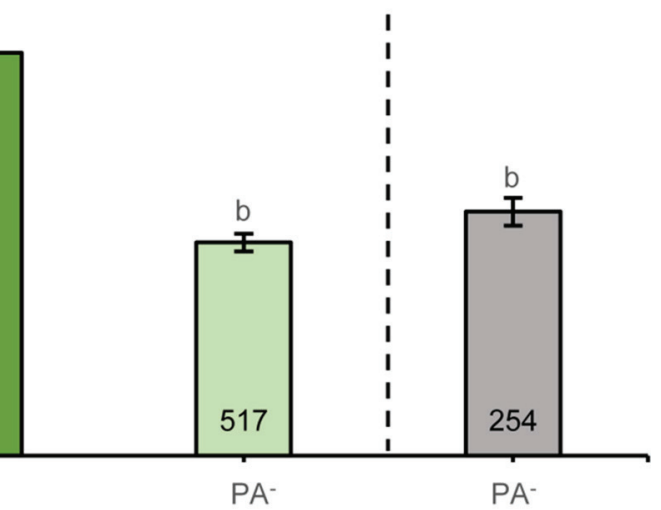

(trp)

(no trp)

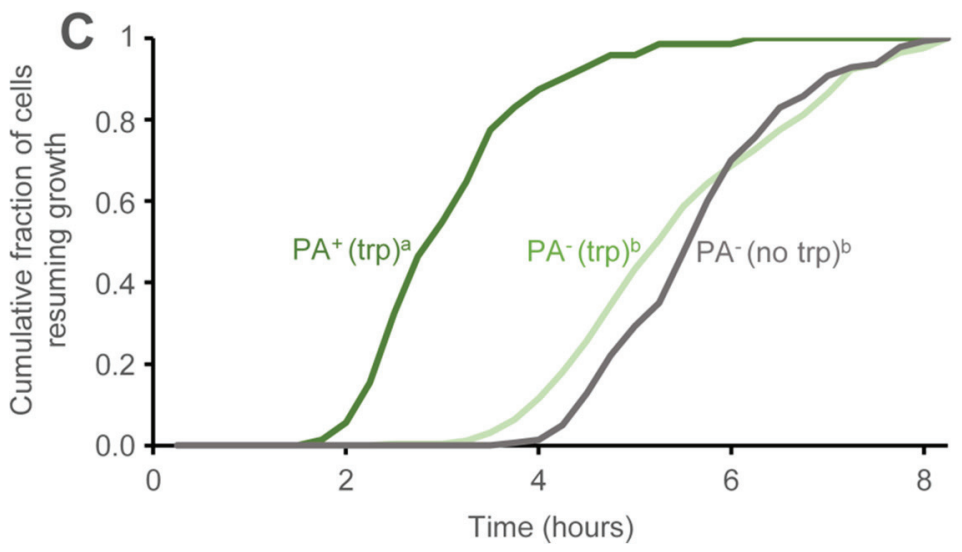

FIG 9 Tryptophan-induced ( $1 \mathrm{mM}$ for $2 \mathrm{~h}$ ) and noninduced exponential-phase founder cells of MG1655 ibpA-msfGFP tna $A^{\Delta 106}$ were grown for ca. 3 generations on $A B$ agarose pads in the absence of tryptophan induction. After heat treatment $\left(53.5^{\circ} \mathrm{C}\right.$ for $\left.15 \mathrm{~min}\right)$ of the resulting microcolonies, survival probability, and lag time of PA-bearing and PA-free cells were determined and compared. (A) Representative phase contrast, epifluorescence (reporting the asymmetrically segregated IbpA-msfGFP labeled PA indicated by the white arrow) and superimposed images of MG1655 ibpA-msfGFP tnaA 106 microcolonies resulting from either a tryptophan-induced (trp; upper panel) or noninduced (no-trp; lower panel) founder cell. Scale bar corresponds to $2 \mu \mathrm{m}$. (B) Survival probability (i) of PA-bearing $\left(\mathrm{PA}^{+}\right.$; dark green; $\left.n=78\right)$ and PA-free (PA ; light green; $\left.n=517\right)$ siblings within heat-treated MG1655 ibpA-msfGFP tnaA $A^{\Delta 106}$ microcolonies stemming from previously induced (trp) founder cells and (ii) of PA-free (PA; gray; $n=245$ ) siblings within heat-treated MG1655 ibpA-msfGFP tnaA ${ }^{\Delta 106}$ microcolonies stemming from noninduced (no trp) founder cells. Different letters indicate significant differences among the bins according to a generalized linear mixed model with the microcolonies considered 
evolution regime, heat shocks were consistently administered to TSB-grown stationaryphase cultures, the timing of HSP expression seems to have been optimized by incurring folding-compromising mutations in a gene properly expressed toward this state.

This apparent selection for the proper timing of resistance deployment underscores the possible modularity of this adaptive mechanism. Indeed, incurring folding-compromising mutations in a (nonessential) gene that is only expressed during a particular environmental condition that tends to coincide with proteotoxic stress would now provide leverage to preemptively upregulate HSP expression and cellular robustness under such conditions. Furthermore, in terms of fitness cost, the transient induction of HSPs by targetedly sacrificing the folding of a timely expressed protein might outcompete mechanisms that more constitutively upregulate HSPs via upstream regulatory mutations (e.g., rpoH mutants) or a more general attenuation of folding fidelity (e.g., ribosome mutants). In fact, it was most recently shown that synthetically engineered $E$. coli mutants with increased proteome-wide mistranslation rates (through an errorprone ribosome [37 or depleted levels of cellular tRNA [38]) displayed constitutively upregulated HSP levels and concurrent heat resistance, although indeed, often at the cost of reduced protein synthesis and growth (38). Additionally, mistranslation inherently imposes a proteome-wide burden on the cell to deal with a potentially nonfunctional quasi-species of protein, while limiting that burden to a single protein would naturally be more beneficial.

For some folding-compromised TnaA variants, the specifics of aggregation seem to result in persistent PAs that become asymmetrically inherited for multiple generations (Fig. 9A). This is of interest since PA inheritance itself has most recently been linked to beneficial features in both Saccharomyces cerevisiae (39) and E. coli (18) model systems. In fact, in agreement with our observations regarding heat-induced PAs in E. coli (18), asymmetric cross-generational segregation of ancestral TnaA-PAs leads to a subpopulation of $\mathrm{PA}^{+}$siblings endowed with clearly improved cellular robustness in terms of higher survival frequency and faster resuscitation speed in response to a heat shock (Fig. 9B and C). Unlike cells experiencing protein aggregation, cells inheriting ancestral (i.e., preformed/established) PAs do not display increased expression of HSPs, although their heat resistance might actually stem from coinheritance of PA-associated HSPS that could fortify the $\mathrm{PA}^{+}$cell (18). While the underlying mechanism of this phenomenon is still elusive, this additional (longer-term) benefit of protein aggregation might nevertheless also constitute a supplementary selectable advantage that shaped the actual nature of some TnaA-variants and the stability of the resulting TnaA-PAs. Interestingly, and in contrast to the faster resuscitation displayed in this study, intracellular PAs have recently also been correlated with dormancy and persistence, indicating that we are currently still scratching the surface with regard to PA physiology $(8,40)$.

Finally, since our data indicate that erosive folding-compromising mutations in proteins can be considered selectable gain-of-function mutations, it is likely that such mutations have left currently unrecognized evolutionary watermarks in the protein sequence space. Indeed, next to their structural or enzymatic cellular function, the evolution of (some) proteins might also have been instructed by their (conditional) folding instability and resulting capacity to boost HSP expression. Likewise, expression patterns of folding-compromised proteins might become tuned to those conditions most in need of higher HSP levels.

FIG 9 Legend (Continued)

random factors (Tukey HSD post hoc test, $P$ value $\leq 0.05$ ). Error bars indicate bootstrapped estimates of the standard error of the mean fraction of surviving cells. (C) Cumulative resuscitation time distributions (i) of surviving PA-bearing ( $\mathrm{PA}^{+}$; dark green; $n=71$ ) and PA-free (PA ; light green; $n=249)$ siblings within heat-treated MG1655 ibpA-msfGFP tnaA $A^{\Delta 106}$ microcolonies stemming from previously induced (trp) founder cells and (ii) of surviving PA-free (PA ; gray; $n=140$ ) siblings within heat-treated MG1655 ibpA-msfGFP tnaA $A^{\Delta 106}$ microcolonies stemming from noninduced (no trp) founder cells. Different letters in superscript indicate significant differences $(P$ value $\leq 0.05)$ among the bins (Kolmogorov-Smirnov tests followed by Bonferroni correction). For panels B and $C$, data were pooled from three independent experiments. 
In summary, our results reveal a new paradigm in bacterial adaptive physiology in which mutations compromising the folding stability of specific proteins can counterintuitively have a selective advantage because of their subtle upregulating effect on cellular HSP levels. The finding that folding-compromising mutations can actually be positively selected for and are not per se loss-of-function mutations can change our view on the evolution of protein sequences.

\section{MATERIALS AND METHODS}

Bacterial strains and growth conditions. The bacterial strains and plasmids used in this study are listed in Table S1, and primers are listed in Table S2. Escherichia coli K-12 strain MG1655 was used as the main background throughout this study. For liquid culturing of bacteria, either tryptone soy broth (TSB; Oxoid, Basingstoke, UK), lysogeny broth according to Lennox (LB), or AB medium (supplemented with $10 \mu \mathrm{g} / \mathrm{ml}$ thiamine, $25 \mu \mathrm{g} / \mathrm{ml}$ uracil, and $1 \%$ Casamino Acids) was used as indicated. AB medium (with the above-mentioned supplements) was also used as solid medium to make agarose pads intended for time-lapse microscopy by the addition of $2 \%$ agarose (Eurogentec, Seraing, Belgium). In the case of single time point microscopy snapshots, $0.85 \% \mathrm{KCl}$ agarose pads ( $2 \%$ agarose) were used. Incubation for cell growth was always done at $37^{\circ} \mathrm{C}$, except for the appropriate times during strain construction when growth at $30^{\circ} \mathrm{C}$ or $42^{\circ} \mathrm{C}$ was required. Liquid cultures were incubated aerobically with shaking (250 rpm) in tubes containing $4 \mathrm{ml}$ of medium. Stationary-phase cultures were obtained by ca. $16 \mathrm{~h}$ of growth, while exponential-phase cultures were obtained by diluting stationary-phase cultures $1 / 100$ or $1 / 1,000$ in fresh medium and allowing growth for ca. 3 to $5 \mathrm{~h}$ (depending on the dilution and the medium used).

When appropriate, the following chemicals were added to the medium at the indicated final concentrations: $100 \mu \mathrm{g} / \mathrm{ml}$ ampicillin (Fisher Scientific, Pittsburgh, PA, USA), $50 \mu \mathrm{g} / \mathrm{ml}$ kanamycin (Applichem, Darmstadt, Germany), $20 \mu \mathrm{g} / \mathrm{ml}$ tetracycline (Applichem), 0.2\% L-arabinose (Acros Organics, Geel, Belgium), 1 to $1.25 \mathrm{mM}$ L-tryptophan (Acros Organics), and $100 \mu \mathrm{M}$ isopropyl $\beta$-D-1-thiogalactopyranoside (IPTG; Acros Organics).

Construction of mutant strains. Mutant alleles (i.e., $\operatorname{tna}^{K 270 A}, \operatorname{tna}^{\Delta 106}, \operatorname{tna}^{259 f s}$, and $r p o H^{154 T}$ ) were exchanged with the corresponding MG1655 wild-type alleles using a previously described two-step process of selection and counterselection (41). For the thaA $A^{\Delta 106}$ and tha $A^{259 f s}$ alleles, the gene was first replaced by an amplicon containing the tet $A-s a c B$ marker prepared on $E$. coli XTL298 using the primers $\mathrm{P} 1$ and P2 (Table S2). In the following step, counterselection against the tet $A$-sac $B$ cassette was used to replace it with a tnaA amplicon obtained on the thaA spontaneous mutants (MT3 and $\mathrm{H} 17$ ) using primers P3 and P4 (Table S2). The tna $A^{K 270 A}$ allele was constructed by placing a tetA-sacB amplicon (obtained with primers P2 and P6; Table S2) immediately downstream of the 270 codon, and the desired mutation was synthetically incorporated in the upstream primer used to obtain the amplicon for counterselection (P7; Table S2). For the point mutation located in the $r p o H$ allele, the tetA-sacB cassette (obtained using primers P10 and P11) was inserted downstream of the gene since it is important for proper cell fitness $(42,43)$, and the clones lacking the cassette after the counterselection step (with an amplicon obtained with primers P12-P13 on a laboratory strain harboring the $r p o H^{154 T}$ allele [unpublished data]) were screened for the presence of the mutation by sequencing.

The in-frame deletion of thaA was performed according to the method of Datsenko and Wanner (44). Briefly, an amplicon prepared with primers P8 and P9 on pKD13 (containing the kanamycin resistance cassette) was recombineered in-frame after the start codon of the target gene of a pKD46equipped strain. The kanamycin resistance gene was flanked by frt sites, enabling it to be excised by transiently equipping the strain with the plasmid pCP20 (expressing the Flp site-specific recombinase [45]). An in-frame deletion of lacY was made in a similar fashion by creating an amplicon on MG1655 lacY::frt-nptl-frt (18) using primers P27 and P28.

To construct chromosomal C-terminal transcriptional fusions of htpG or tnaA with msfGFP, the msfGFP-frt-nptl-frt cassette was amplified from the previously described pDHL1029 plasmid (46) using primers P15 and P16 (htpG) or P3 and P4 (tnaA). Similarly, to create a C-terminal transcriptional fusion of DnaK with $m S$ carlet-l, the $m S$ carlet-I-frt-nptl-frt cassette was amplified from pBAM1-Tn5-mScarlet-I using primers P35 and P36. Subsequent recombineering into the correct locus was achieved by lambda Redmediated recombination using pKD46 (44). The amplicon was placed $5 \mathrm{bp}$ after the stop codon of the gene of interest, creating an artificial operon and ensuring cotranscription. A strong synthetic ribosome binding site (BBa_B0034; sequence AAAGAGGAGAA [47]) was used to facilitate msfGFP or mScarlet-I expression. Subsequently, the frt-flanked kanamycin resistance cassette was excised by site-specific recombination by transiently equipping the strain with plasmid pCP20. Equipping strains with the previously described chromosomal translational ibpA-msfgfp fusion (18) was achieved in similar fashion. The amplicon for recombineering was obtained from MG1655 ibpA-msfGFP-frt-nptl-frt (18) using primers P19 and P20, followed by the above-mentioned steps to achieve the desired translational fusion.

All constructed mutants were verified by $P C R$, using primers that anneal upstream and downstream of the engineered locus (Table S2). Subsequently, the mutated genes of interest were confirmed by sequencing (Macrogen, Amsterdam, the Netherlands).

Construction of plasmids. A set of pTrc99A plasmids capable of the IPTG-inducible expression of different tnaA alleles was constructed by first making an amplicon of the corresponding tna $A$ allele with primer pair P23-P24. These primers amplified the thaA open reading frame and introduced Ncol and Xbal restriction sites to the ends of the amplicon. Subsequently, digestion of both the pTrc99A vector and the amplicons with $\mathrm{Ncol}$ and $\mathrm{Xbal}$ allowed the directed ligation of the amplicon into the vector. 
Plasmid pBAM1-Tn5-mScarlet-I was constructed by amplifying the pBAM1-Tn5 backbone from pBAM1-Tn5-mVenus (48) (using primers P29 and P30) and creating an amplicon of the mScarlet-l gene with primers P31 and P32. These amplicons were subsequently assembled into the pBAM1-Tn5mScarlet-I plasmid using Gibson assembly (New England BioLabs, Ipswich, MA, USA).

All constructed plasmids were confirmed by amplicon sequencing of the engineered sites (Macrogen) and introduced into the target host strains by electroporation and selection for antibiotic resistance encoded by the plasmid.

Heat treatment of liquid cultures. For heat treatment, cultures were harvested by centrifugation $(6,000 \times g, 5 \mathrm{~min})$ and resuspended in an equal volume of $0.85 \% \mathrm{KCl}$. Next, $50 \mu \mathrm{l}$ of the resuspended culture was transferred aseptically to a sterile PCR tube and heat-treated for $15 \mathrm{~min}$ in a T3000 thermocycler (Biometra, Göttingen, Germany) at the indicated temperatures. Additionally, unstressed control cultures were kept at room temperature for the duration of the heat treatment. Samples were aseptically retrieved from the PCR tubes and subsequently used to determine survival, as described below.

Population level determination of viability. Heat-stressed and unstressed bacterial cultures were serially diluted in $0.85 \% \mathrm{KCl}$ and subsequently spotted $(5 \mu \mathrm{l})$ onto tryptone soy agar (TSA; Oxoid) or LB agar (corresponding to the initial growth conditions unless indicated otherwise) as previously described (49). In the case of growth in AB medium, cells were recovered on either TSA or LB agar plates, as indicated. After $24 \mathrm{~h}$ of incubation at $37^{\circ} \mathrm{C}$, the colonies in spots containing between 5 and 50 colonies were counted to determine the $\mathrm{CFU} / \mathrm{ml}$, so that the limit of quantification was $1,000 \mathrm{CFU} / \mathrm{ml}$. Subsequently, the logarithmic reduction factor, $\log _{10}\left(N_{0} / N\right)$, was determined, in which $N_{0}$ and $N$ represent the $C F U / m$ l prior to and after heat treatment, respectively.

Selection of heat-resistant mutants by directed evolution. Heat-resistant mutants of MG1655 $\triangle$ rpoS ibpA-msfGFP were obtained by repeatedly subjecting independent overnight TSB cultures to increasingly severe 15 -min heat shocks (from $51^{\circ} \mathrm{C}$ to $55^{\circ} \mathrm{C}$ with $0.5^{\circ} \mathrm{C}$ increments) with intermittent resuscitation and outgrowth of the survivors. After each heat shock, the heat-treated samples were diluted $1 / 100$ in fresh TSB and regrown for $23 \mathrm{~h}$ at $37^{\circ} \mathrm{C}$ before the next round of heat treatment. Following nine cycles of selection, a single surviving clone from each culture was purified on TSA and tested for increased heat resistance at $55^{\circ} \mathrm{C}(15 \mathrm{~min})$, as described above. Additionally, a number of independent lineages were subjected to the same regime in the absence of heat stress in order to determine the potential selective effect of serially passing through TSB.

Whole-genome sequencing (WGS). Genomic DNA was isolated from overnight LB cultures using the GeneJET genomic DNA purification kit (Thermo Fisher Scientific, Waltham, MA, USA), after which 150-bp paired-end libraries were prepared using the Flex library prep kit (Illumina, San Diego, CA, USA) and the Nextra DNA CD index kit (Illumina). Sequencing was performed with an Illumina MiniSeq sequencer and analyzed with CLC Genomics Workbench (Qiagen, Hilden, Germany). The sequencing reads were trimmed and mapped to the reference genome (MG1655) and analyzed for single nucleotide polymorphisms (SNPs), indels, and structural variants.

Determining indole concentration. Indole concentration was determined based on a previously described method (50). Stationary-phase TSB cultures were harvested $(6,000 \times g, 5 \mathrm{~min})$, and the supernatant was transferred to a new recipient, to which $300 \mu$ l of Kovac's reagent (Sigma-Aldrich, Saint Louis, MO, USA) was added. After $2 \mathrm{~min}$ of incubation, $20 \mu \mathrm{l}$ from the top was transferred to a recipient containing $100 \mu \mathrm{l}$ of $37 \% \mathrm{HCl}$ and $300 \mu \mathrm{l}$ of $100 \%$ ethanol. The absorbance of $200 \mu \mathrm{l}$ of this mixture was measured at $550 \mathrm{~nm}$ in a microtiter plate using a Multiskan RC instrument (Thermo LabSystems, Vantaa, Finland). Indole concentration was estimated using a calibration curve obtained by the absorbance values at $550 \mathrm{~nm}$ after adding 0 to $400 \mu \mathrm{M}$ indole (Applichem) to stationary-phase cultures of MG1655 $\Delta$ tnaA.

Protein extraction. For protein extraction, $40 \mathrm{ml}$ of an exponential-phase culture was harvested $\left(6,000 \times \mathrm{g}, 10 \mathrm{~min}, 4^{\circ} \mathrm{C}\right)$ and resuspended in $10 \mathrm{mM}$ Tris- $\mathrm{HCl}(\mathrm{pH} 7.5)$ containing $150 \mathrm{mM} \mathrm{NaCl}$ and $5 \mathrm{mM}$ $\beta$-mercaptoethanol. The resuspension volume was normalized based on the initial optical density at $600 \mathrm{~nm}$. To break the cells, resuspensions were sonicated (VCX30, Sonics \& Materials, Newtown, CT, USA) on ice for a total of $2 \mathrm{~min}$ (6 pulses of $20 \mathrm{~s}$ at $30 \%$ amplitude with 20-s pauses in between). To separate the cellular protein content in soluble and insoluble fractions, the lysate was centrifuged at 13,000 $\times g$ for $15 \mathrm{~min}$ at $4^{\circ} \mathrm{C}$. The supernatant was collected and retained as the soluble fraction. The pellet (insoluble fraction) was subsequently washed three times $\left(13,000 \times g\right.$ for $15 \mathrm{~min}$ at $\left.4^{\circ} \mathrm{C}\right)$ with buffer $X(50 \mathrm{mM} \mathrm{HEPES}$ [pH 7.5], $300 \mathrm{mM} \mathrm{NaCl}, 5 \mathrm{mM} \beta$-mercaptoethanol, $0.1 \mathrm{mM}$ EDTA, and protease inhibitor cocktail [Sigma-Aldrich]). The samples were further purified by three washing steps $\left(13,000 \times g\right.$ for $15 \mathrm{~min}$ at $4^{\circ} \mathrm{C}$ ) with buffer $Y$ (same as buffer $X$ but without protease inhibitor and supplemented with $0.8 \%$ [vol/vol] Triton $X_{-} 100$ and $0.1 \%$ sodium deoxycholic acid), with sonicating after every wash step for $10 \mathrm{~s} \mathrm{(5} \mathrm{pulses} \mathrm{of} 2 \mathrm{~s}$ at $30 \%$ amplitude with 2-s pauses in between). Finally, samples were resuspended in buffer Z (50 mM HEPES [pH 7.5], 8.0 M urea), heat-treated $\left(10 \mathrm{~min}, 90^{\circ} \mathrm{C}\right)$, and used for SDS-PAGE (12\% gel, $20 \mu \mathrm{l}$ per well). After SDS-PAGE, gels were stained with Coomassie brilliant blue G-250 as previously described (51).

Western blotting. Cells were harvested by centrifugation at 4,000 $\times g$ for $30 \mathrm{~min}$, washed first with physiological water and then washed with $10 \mathrm{ml}$ of buffer A (50 mM HEPES [pH 7.5], $300 \mathrm{mM} \mathrm{NaCl}, 5 \mathrm{mM}$ $\beta$-mercaptoethanol, and $1.0 \mathrm{mM}$ EDTA). Pellets were finally resuspended in $20 \mathrm{ml}$ of buffer B (buffer A supplemented with a protease inhibitor cocktail). Cells were broken with a Glen Creston cell homogenizer $\left(20,000\right.$ to $\left.25,000 \mathrm{lb} / \mathrm{in}^{2}\right)$ and additional sonication (Branson digital sonifier $50 / 60 \mathrm{HZ}$ ) on ice with alternating 2 -min cycles ( 15 pulses at $50 \%$ power with 30-s pauses on ice, until completing 2 min of total sonication time).

For protein separation, SDS-PAGE was performed using Any kD precast gels (Bio-Rad, Hercules, CA, USA), after which, proteins were transferred on a nitrocellulose membrane (Trans-Blot Turbo Mini 0.2$\mu \mathrm{m}$ nitrocellulose transfer packs; Bio-Rad) with the Trans-Blot Turbo transfer system (Bio-Rad). 
Afterward, the membrane was incubated for $1 \mathrm{~h}$ in $5 \%$ nonfat dry milk in TBST (Tris-buffered saline with Tween 20, pH 8.0) and then stained overnight with the primary antibodies, i.e., monoclonal mouse antiDnaK ( $1 \mu \mathrm{g} / \mathrm{ml}$; Aviva Systems Biology, San Diego, CA) or anti-GroEL ( $1 \mu \mathrm{g} / \mathrm{ml}$; Abcam, Cambridge, UK). The membranes were washed three times for $10 \mathrm{~min}$ with $0.5 \%$ Tween 20 in phosphate-buffered saline (PBS) buffer and stained with the secondary antibody for $2 \mathrm{~h}$ (goat anti-mouse IgG HRP; Abcam). After washing, horseradish peroxidase (HRP) was detected with an enhanced chemiluminescence system (ChemiDoc Imaging System; Bio-Rad), and band intensity was quantified with the Image Lab software (Bio-Rad). The density of each band after background subtraction was expressed in fold change compared to the parental $\Delta r p o S$ strain. Western blot (WB) analysis was performed four times on four independent stationary-phase cultures of each strain.

Liquid chromatography and mass spectrometry (LC-MS/MS). For protein identification, gel pieces were extracted based on the protocol of Shevchenko et al. (52). Digestion was performed overnight at $37^{\circ} \mathrm{C}$. Samples were desalted using Pierce $\mathrm{C} 18$ solid-phase extraction columns according to the manufacturer's instructions (Thermo Scientific) and dried in a SpeedVac until dry and dissolved in $20 \mu \mathrm{l}$ 5\% acetonitrile $(\mathrm{ACN})$ and $0.1 \%$ formic acid. The digested and desalted samples were diluted 10 times and injected $(5 \mu \mathrm{l})$ and separated on an Ultimate 3000 UPLC system (Dionex, Thermo Scientific) equipped with an Acclaim PepMap100 precolumn $\left(C_{18}\right.$ particle size, $3 \mu \mathrm{m}$; pore size, $100 \AA$; diameter, $0.075 \mathrm{~mm}$; length, $20 \mathrm{~mm}$; Thermo Scientific) and a $C_{18}$ PepMap rapid-separation liquid chromatography (RSLC) system (particle size, $2 \mu \mathrm{m}$; pore size, $100 \AA$; diameter, $50 \mu \mathrm{m}$; length, $150 \mathrm{~mm}$; Thermo Scientific) using a linear gradient $(0.300 \mu \mathrm{l} / \mathrm{min})$. The composition of buffer $\mathrm{A}$ is pure water containing $0.1 \%$ formic acid. The composition of buffer $\mathrm{B}$ is pure water containing $0.08 \%$ formic acid and $80 \%$ acetonitrile. The fraction 0 to $4 \%$ of buffer B ( $80 \%$ ACN, $0.08 \%$ formic acid) increased from 0 to $4 \%$ in 3 min, from 4 to $10 \%$ B in $12 \mathrm{~min}$, from 10 to $35 \%$ in $20 \mathrm{~min}$, from 35 to $65 \%$ in $5 \mathrm{~min}$, and from 65 to $95 \%$ in $1 \mathrm{~min}$ and stayed at $95 \%$ for $10 \mathrm{~min}$. The fraction of buffer B, decreased from 95 to $5 \%$ in $1 \mathrm{~min}$ and stayed at $5 \%$ for 10 min. The Orbitrap Elite mass spectrometer (Thermo Scientific) was operated in positive ion mode with a nanospray voltage of $2.1 \mathrm{kV}$ and a source temperature of $250^{\circ} \mathrm{C}$. Pierce LTQ Velos ESI positive ion calibration mix (catalog no. 88323, Thermo Scientific) was used as an external calibrant. The instrument was operated in data-dependent acquisition mode with a survey MS scan at a resolution of 70,000 (full width at half maximum [fwhm]) for the mass range of $\mathrm{m} / \mathrm{z} 400$ to 1,600 for precursor ions, followed by MS/MS scans of the top 10 most intense peaks with $+2,+3,+4$, and +5 charged ions above a threshold ion count of $16,0001 \mathrm{e}+6$ at 17,500 resolution (full width at half maximum [fwhm]) using a normalized collision energy of $25 \mathrm{eV}$ with an isolation window of $3.0 \mathrm{~m} / \mathrm{z}$, Apex trigger of 5 to $15 \mathrm{~s}$, and dynamic exclusion of $10 \mathrm{~s}$. All data were acquired with Xcalibur 3.1.66.10 software (Thermo Scientific).

Protein identification. Tandem mass spectra were extracted using Progenesis $v 4.1$ (Nonlinear Dynamics, UK). All MS/MS samples were analyzed using Mascot v 2.2.06 (Matrix Science, London, UK) and X! Tandem v X! (The Global Proteome Machine [GPM], thegpm.org; Tandem Alanine [2017.2.1.4]). Mascot was set up to search the uniprot_ecolimax_database $(282,225$ entries, where the mutated sequences have been added) assuming the digestion enzyme trypsin. X!, Tandem was set up to search a reverse concatenated uniprot_ecolimax_database (unknown version, 564,448 entries). Mascot and X! Tandem were searched with a fragment ion mass tolerance of $0.20 \mathrm{Da}$ and a parent ion tolerance of 12 ppm and 2 possible miscleavages. Carbamidomethyl of cysteine was specified in Mascot and X! Tandem as a fixed modification. Deamidation of asparagine and glutamine and oxidation of methionine were specified in Mascot as variable modifications. Glu->pyro-Glu of the $\mathrm{N}$ terminus, ammonia-loss of the $\mathrm{N}$ terminus, gln->pyro-Glu of the $\mathrm{N}$ terminus, deamidation of asparagine, and glutamine and oxidation of methionine were specified in $\mathrm{X}$ ! Tandem as variable modifications.

Scaffold v 4.11.0 (Proteome Software, Inc., Portland, OR) was used to validate MS/MS-based peptide and protein identifications. Peptide identifications were accepted if they could be established at greater than $97.0 \%$ probability by the Peptide Prophet algorithm (53) with Scaffold delta-mass correction. Protein probability was assigned by the Protein Prophet algorithm (54).

Time-lapse fluorescence microscopy (TLFM). For TLFM, appropriate dilutions of cell cultures were transferred to agarose pads containing the appropriate medium on a microscopy slide and covered with a cover glass attached to a $125-\mu$ l Gene Frame (Thermo Fisher Scientific) to hold the cover glass on the microscopy slide. TLFM was performed on a Ti-Eclipse inverted microscope (Nikon, Champigny-surMarne, France) equipped with a $\times 60$ Plan Apo $\lambda$ oil objective, a TI-CT-E motorized condenser, and a Nikon DS-Qi2 camera. Green fluorescent protein (GFP) was imaged using a quad-edge dichroic (395/ $470 / 550 / 640 \mathrm{~nm}$ ) and a fluorescein isothiocyanate (FITC) single emission filter. A SpecraX LED illuminator (Lumencor, Beaverton, OR, USA) was used as the light source, using the 470/24 excitation filter. Temperature was controlled with an cage incubator (Okolab, Ottaviano, Italy).

Images were acquired using NIS-Elements software (Nikon), and the resulting pictures were further handled with the open source software ImageJ. The average cellular fluorescence of cells was determined using the open source software llastik (55), which was trained to robustly identify and segment bacterial cells and exclude debris and out-of-focus cells. Background fluorescence was subtracted using NIS-elements software.

Single-cell determination of viability and resuscitation time. Cells were placed on agarose pads on a microscopy slide, as described above, and monitored with TLFM during growth for $2 \mathrm{~h}$ at $37^{\circ} \mathrm{C}$ (approximately 3 generations). Subsequently, the $X Y$ coordinates of the observed microcolonies were determined with NIS-Elements software, and the slide was subjected to a semilethal heat shock by taping the slide to the lid of a thermocycler set to the appropriate temperature $\left(53.5^{\circ} \mathrm{C}, 15 \mathrm{~min}\right)$. After the heat shock, the locations of the heat-exposed microcolonies were traced back using the XY coordinates, and the cells were further monitored for $8 \mathrm{~h}$ to determine survival and lag time. Subsequently, the 
number of cells surviving the heat shock in microcolonies was determined by monitoring cells with TLFM. Cells were marked as surviving cells when they were observed to grow and divide within $8 \mathrm{~h}$ after the heat treatment. The time of the first binary fission after heat treatment was used as a proxy for resuscitation time. Cells were binned according to whether they had an inclusion body by visual determination or a fluorescent IbpA-msfGFP focus before heat treatment.

Thermodynamic stability calculations of TnaA variants. Based on the TnaA sequence of the $E$. coli K-12 MG1655 strain used throughout this study (protein and nucleotide sequences in Data Set S2), all possible single amino acid substitutions that can theoretically result from a single nucleotide mutation were generated, resulting in a list of 2,765 variants. In addition, naturally occurring substitutions were gathered from a set of sequences orthologous to $E$. coli K-12 TnaA from the OMA (Orthologous Matrix) database (56), resulting in 218 sequences after removing outliers with internal indels over 10 residues compared to the reference sequence (Data Set S3). A substitution was classified as naturally occurring if it occurs in at least one of the TnaA orthologues. The effects of these substitutions and the heat-selected substitutions on TnaA thermodynamic stability were calculated and compared using FoldX3.0 with default settings (29) on the pdb-structure 2oqx (57).

Statistical analysis. Statistical analyses (analysis of variance [ANOVA], Tukey honestly significant difference [HSD] post hoc test, $t$ test, Kolmogorov-Smirnov (KS) test, generalized linear mixed models, Bonferroni correction, bootstrapping [and the appropriate tests to test for underlying assumptions]), were carried out using the open source software R (R Core Team, 2020) (60). Differences were regarded as significant when the $P$ value was $\leq 0.05$. Means and the corresponding standard errors were typically calculated over three independent experiments.

To estimate the standard error of the surviving cellular fractions in Fig. 9B, the original sample size was bootstrapped (sampled with replacement) 10,000 times to calculate the mean fractions of surviving cells. This allowed the calculation of a bootstrapped estimation of the standard error of the mean fraction of surviving cells by determining the standard deviation of the bootstrapped means.

\section{SUPPLEMENTAL MATERIAL}

Supplemental material is available online only.

DATA SET S1, XLSX file, $0.01 \mathrm{MB}$.

DATA SET S2, DOCX file, $0.01 \mathrm{MB}$.

DATA SET S3, XLSX file, $0.9 \mathrm{MB}$.

FIG S1, TIF file, 1.9 MB.

FIG S2, TIF file, 2.5 MB.

FIG S3, TIF file, 1.9 MB.

FIG S4, TIF file, 1.8 MB.

FIG S5, TIF file, 2.5 MB.

TABLE S1, DOCX file, $0.02 \mathrm{MB}$.

TABLE S2, DOCX file, $0.01 \mathrm{MB}$.

\section{ACKNOWLEDGMENTS}

We thank Aylin Aytek for her help in strain construction, Kusay Arat for his aid in the mass spectrometry analysis, and Sander Govers for critical reading of the manuscript.

This work was supported by a doctoral fellowship (11B0519N to J.M.), a postdoctoral fellowship (12P9818N to E.G), grants (G0C7118N and G0D8220N to A.A.) from the Research Foundation-Flanders (FWO-Vlaanderen), and a postdoctoral fellowship (PDM/20/ 118 to J.M.) from the KU Leuven Research Fund. The Switch Laboratory was supported by the Flanders institute for biotechnology (VIB), the University of Leuven and its Industrieel Onderzoeksfonds, and the Funds for Scientific Research Flanders (FWO).

\section{REFERENCES}

1. Raso J, Barbosa-Cánovas GV. 2003. Nonthermal preservation of foods using combined processing techniques. Crit Rev Food Sci Nutr 43:265-285. https://doi.org/10.1080/10408690390826527.

2. Ross AIV, Griffiths MW, Mittal GS, Deeth HC. 2003. Combining nonthermal technologies to control foodborne microorganisms. Int J Food Microbiol 89:125-138. https://doi.org/10.1016/S0168-1605(03)00161-2.

3. Mccoy LS, Xie Y, Tor Y. 2011. Antibiotics that target protein synthesis. Wiley Interdiscip Rev RNA 2:209-232. https://doi.org/10.1002/wrna.60.

4. Van Acker H, Coenye T. 2017. The role of reactive oxygen species in antibiotic-mediated killing of bacteria. Trends Microbiol 25:456-466. https://doi .org/10.1016/j.tim.2016.12.008.
5. Imlay JA. 2019. Where in the world do bacteria experience oxidative stress? Environ Microbiol 21:521-530. https://doi.org/10.1111/1462-2920 .14445 .

6. Winterbourn CC, Kettle AJ, Hampton MB. 2016. Reactive oxygen species and neutrophil function. Annu Rev Biochem 85:765-792. https://doi.org/ 10.1146/annurev-biochem-060815-014442.

7. Schramm FD, Schroeder K, Jonas K. 2020. Protein aggregation in bacteria. FEMS Microbiol Rev 44:54-72. https://doi.org/10.1093/femsre/fuz026.

8. Govers SK, Dutré P, Aertsen A. 2014. In vivo disassembly and reassembly of protein aggregates in Escherichia coli. J Bacteriol 196:2325-2332. https://doi.org/10.1128/JB.01549-14. 
9. Gottesman S, Wickner S, Maurizi MR. 1997. Protein quality control: triage by chaperones and proteases. Genes Dev 11:815-823. https://doi.org/10 1101/gad.11.7.815.

10. Mogk A, Huber D, Bukau B. 2011. Integrating protein homeostasis strategies in prokaryotes. Cold Spring Harb Perspect Biol 3:1-19. https://doi .org/10.1101/cshperspect.a004366.

11. Straus D, Walter W, Gross CA. 1987. The heat shock response of E. coli is regulated by changes in the concentration of sigma 32. Nature 329:348-351. https://doi.org/10.1038/329348a0.

12. Guisbert E, Yura T, Rhodius VA, Gross CA. 2008. Convergence of molecular, modeling, and systems approaches for an understanding of the Escherichia coli heat shock response. Microbiol Mol Biol Rev 72:545-554. https://doi.org/10.1128/MMBR.00007-08.

13. Morita MT, Kanemori M, Yanagi H, Yura T. 2000. Dynamic interplay between antagonistic pathways controlling the $\sigma 32$ level in Escherichia coli. Proc Natl Acad Sci U S A 97:5860-5865. https://doi.org/10.1073/pnas .080495197.

14. Guisbert E, Herman C, Lu CZ, Gross CA. 2004. A chaperone network controls the heat shock response in E. coli. Genes Dev 18:2812-2821. https:// doi.org/10.1101/gad.1219204.

15. Wang Z, Fang Y, Zhi S, Simpson DJ, Gill A, McMullen LM, Neumann NF, Gänzle NG. 2020. The locus of heat resistance confers resistance to chlorine and other oxidizing chemicals in Escherichia coli. Appl Environ Microbiol 86:e02123-19. https://doi.org/10.1128/AEM.02123-19.

16. Nguyen SV, Harhay GP, Bono JL, Smith TPL, Harhay DM. 2017. Genome sequence of the thermotolerant foodborne pathogen Salmonella enterica serovar Senftenberg ATCC 43845 and phylogenetic analysis of loci encoding increased protein quality control mechanisms. mSystems 2: e00190-16. https://doi.org/10.1128/mSystems.00190-16.

17. Guragain M, Smith GE, King DA, Bosilevac JM. 2020. Prevalence of extreme heat-resistant Gram-negative bacteria carried by U.S. cattle at harvest. J Food Prot 83:1438-1443. https://doi.org/10.4315/JFP-20-103.

18. Govers SK, Mortier J, Adam A, Aertsen A. 2018. Protein aggregates encode epigenetic memory of stressful encounters in individual Escherichia coli cells. PLoS Biol 16:e2003853-40. https://doi.org/10.1371/ journal.pbio.2003853.

19. Gayán E, Cambré A, Michiels CW, Aertsen A. 2017. RpoS-independent evolution reveals the importance of attenuated CAMP/CRP regulation in high hydrostatic pressure resistance acquisition in E. coli. Sci Rep 7:8600. doi:https://doi.org/10.1038/s41598-017-08958-z.

20. Li G, Young KD. 2015. A new suite of tnaA mutants suggests that Escherichia coli tryptophanase is regulated by intracellular sequestration and by occlusion of its active site. BMC Microbiol 15:14. https://doi.org/10 .1186/s12866-015-0346-3.

21. Li G, Young KD. 2013. Indole production by the tryptophanase TnaA in Escherichia coli is determined by the amount of exogenous tryptophan. Microbiology (Reading) 159:402-410. https://doi.org/10.1099/mic.0.064139-0.

22. Bilezikian JP, Kaempfer ROR, Magasanik B. 1967. Mechanism of tryptophanase induction in Escherichia coli. J Mol Biol 27:495-506. https://doi.org/ 10.1016/0022-2836(67)90054-X.

23. Li G, Young KD. 2014. A cAMP-independent carbohydrate-driven mechanism inhibits tnaA expression and TnaA enzyme activity in Escherichia coli. Microbiology (Reading) 160:2079-2088. https://doi.org/10.1099/mic .0.080705-0.

24. Isaacs H, Chao D, Yanofsky C, Saier MH. 1994. Mechanism of catabolite repression of tryptophanase synthesis in Escherichia coli. Microbiology 140:2125-2134. https://doi.org/10.1099/13500872-140-8-2125.

25. Botsford JL, DeMoss RD. 1971. Catabolite repression of tryptophanase in Escherichia coli. J Bacteriol 105:303-312. https://doi.org/10.1128/jb.105.1 303-312.1971.

26. Sezonov G, Joseleau-Petit D, D'Ari R. 2007. Escherichia coli physiology in Luria-Bertani broth. J Bacteriol 189:8746-8749. https://doi.org/10.1128/JB .01368-07.

27. Yura T, Guisbert E, Poritz M, Lu CZ, Campbell E, Gross CA. 2007. Analysis of $\sigma 32$ mutants defective in chaperone-mediated feedback control reveals unexpected complexity of the heat shock response. Proc Natl Acad Sci U S A 104:17638-17643. https://doi.org/10.1073/pnas.0708819104.

28. Gray VE, Hause RJ, Fowler DM. 2017. Analysis of large-scale mutagenesis data to assess the impact of single amino acid substitutions. Genetics 207:53-61. https://doi.org/10.1534/genetics.117.300064.

29. Schymkowitz J, Borg J, Stricher F, Nys R, Rousseau F, Serrano L. 2005. The FoldX web server: an online force field. Nucleic Acids Res 33:382-388. https://doi.org/10.1093/nar/gki387.
30. Wain J, House D, Parkhill J, Parry C, Dougan G. 2002. Unlocking the genome of the human typhoid bacillus. Lancet Infect Dis 2:163-170. https:// doi.org/10.1016/S1473-3099(02)00225-6.

31. Chant EL, Summers DK. 2007. Indole signalling contributes to the stable maintenance of Escherichia coli multicopy plasmids. Mol Microbiol 63:35-43. https://doi.org/10.1111/j.1365-2958.2006.05481.x.

32. Chimerel C, Field CM, Piñero-Fernandez S, Keyser UF, Summers DK. 2012. Indole prevents Escherichia coli cell division by modulating membrane potential. Biochim Biophys Acta Biomembr 1818:1590-1594. https://doi .org/10.1016/j.bbamem.2012.02.022.

33. Lee HH, Molla MN, Cantor CR, Collins JJ. 2010. Bacterial charity work leads to population-wide resistance. Nature 467:82-86. https://doi.org/10 .1038/nature09354.

34. Vega NM, Allison KR, Khalil AS, Collins JJ. 2012. Signaling-mediated bacterial persister formation. Nat Chem Biol 8:431-433. https://doi.org/10 .1038/nchembio.915.

35. Bommarius B, Anyanful A, Izrayelit $Y$, Bhatt S, Cartwright E, Wang W, Swimm Al, Benian GM, Schroeder FC, Kalman D. 2013. A family of indoles regulate virulence and Shiga toxin production in pathogenic E. coli. PLoS One 8:e54456-16. https://doi.org/10.1371/journal.pone.0054456.

36. Lee J, Jayaraman A, Wood TK. 2007. Indole is an inter-species biofilm signal mediated by SdiA. BMC Microbiol 7:42-15. https://doi.org/10.1186/ 1471-2180-7-42.

37. Evans CR, Fan Y, Ling J. 2019. Increased mistranslation protects E. coli from protein misfolding stress due to activation of a RpoS-dependent heat shock response. FEBS Lett 593:3220-3227. https://doi.org/10.1002/ 1873-3468.13578.

38. Samhita L, Raval PK, Agashe D. 2020. Global mistranslation increases cell survival under stress in Escherichia coli. PLoS Genet 16:e1008654-21. https://doi.org/10.1371/journal.pgen.1008654.

39. Saarikangas J, Barral Y. 2015. Protein aggregates are associated with replicative aging without compromising protein quality control. Elife 4: e06197. https://doi.org/10.7554/eLife.06197.

40. Pu Y, Li Y, Jin X, Tian T, Ma Q, Zhao Z, Lin S-Y, Chen Z, Li B, Yao G, Leake MC, Lo C-J, Bai F. 2019. ATP-dependent dynamic protein aggregation regulates bacterial dormancy depth critical for antibiotic tolerance. Mol Cell 73:143-156. https://doi.org/10.1016/j.molcel.2018.10.022.

41. Li XT, Thomason LC, Sawitzke JA, Costantino N, Court DL. 2013. Positive and negative selection using the tetA-sacB cassette: recombineering and P1 transduction in Escherichia coli. Nucleic Acids Res 41:e204. https://doi .org/10.1093/nar/gkt1075.

42. Baba T, Ara T, Hasegawa M, Takai Y, Okumura Y, Baba M, Datsenko KA, Tomita M, Wanner BL, Mori H. 2006. Construction of Escherichia coli K-12 in-frame, single-gene knockout mutants: the Keio collection. Mol Syst Biol 2:2006.0008. https://doi.org/10.1038/msb4100050.

43. Goodall ECA, Robinson A, Johnston IG, Jabbari S, Turner KA, Cunningham AF, Lund PA, Cole JA, Henderson IR. 2018. The essential genome of Escherichia coli K-12. mBio 9:e02096-17. https://doi.org/10.1128/mBio.02096-17.

44. Datsenko KA, Wanner BL. 2000. One-step inactivation of chromosomal genes in Escherichia coli K-12 using PCR products. Proc Natl Acad Sci U S A 97:6640-6645. https://doi.org/10.1073/pnas.120163297.

45. Cherepanov PP, Wackernagel W. 1995. Gene disruption in Escherichia coli: Tc R and Km R cassettes with the option of Flp-catalyzed excision of the antibiotic-resistance determinant. Gene 158:9-14. https://doi.org/10 .1016/0378-1119(95)00193-A.

46. Ke N, Landgraf D, Paulsson J, Berkmen M. 2016. Visualization of periplasmic and cytoplasmic proteins with a self-labeling protein tag. J Bacteriol 198:1035-1043. https://doi.org/10.1128/JB.00864-15.

47. Elowitz MB, Leibler S. 2000. A synthetic oscillatory network of transcriptional regulators. Nature 403:335-338. https://doi.org/10.1038/35002125.

48. Passaris I, Cambré A, Govers SK, Aertsen A. 2018. Bimodal expression of the Salmonella Typhimurium. Genetics 210:621-635. https://doi.org/10 .1534/genetics.118.300822.

49. Sieuwerts S, De Bok FAM, Mols E, De Vos WM, Van Hylckama Vlieg JET. 2008. A simple and fast method for determining colony forming units. Lett Appl Microbiol 47:275-278. https://doi.org/10.1111/j.1472-765X .2008.02417.x.

50. Gaimster H, Cama J, Hernández-Ainsa S, Keyser UF, Summers DK. 2014. The indole pulse: a new perspective on indole signalling in Escherichia coli. PLoS One 9:e93168. https://doi.org/10.1371/journal.pone.0093168.

51. Candiano G, Bruschi M, Musante L, Santucci L, Ghiggeri GM, Carnemolla B, Orecchia P, Zardi L, Righetti PG. 2004. Blue silver: a very sensitive colloidal Coomassie G-250 staining for proteome analysis. Electrophoresis 25:1327-1333. https://doi.org/10.1002/elps.200305844. 
52. Shevchenko A, Tomas H, Havliš J, Olsen JV, Mann M. 2006. In-gel digestion for mass spectrometric characterization of proteins and proteomes. Nat Protoc 1:2856-2860. https://doi.org/10.1038/nprot.2006.468.

53. Keller AD, Nesvizhskii Al, Kolker E, Aebersold R. 2002. Empirical statistical model to estimate the accuracy of peptide identifications made by MS/ MS and database search. Anal Chem 74:5383-8392. https://doi.org/10 .1021/ac025747h.

54. Nesvizhskii Al, Keller A, Kolker E, Aebersold R. 2003. A statistical model for identifying proteins by tandem mass spectrometry. Anal Chem 75:4646-4658. https://doi.org/10.1021/ac0341261.

55. Sommer C, Strähle C, Köthe U, Hamprecht FA. 2011. Ilastik: interactive learning and segmentation toolkit, $\mathrm{p}$ 230-233. In Proceedings of the eighth IEEE International Symposium on Biomedical Imaging (ISBI). IEEE. https://doi.org/10.1109/ISBI.2011.5872394.

56. Altenhoff AM, Škunca N, Train C-M, Sueki A, Piližota I, Gori K, Bartlomiej T, Müller S, Redestig H, Gonnet GH, Dessimoz C. 2015. The OMA orthology database in 2015: function predictions, better plant support, synteny view and other improvements. Nucleic Acids Res 43:240-249. https://doi .org/10.1093/nar/gku1158.

57. Tsesin N, Kogan A, Gdalevsky GY, Himanen JP, Cohen-Luria R, Parola AH, Goldgur Y, Almog O. 2007. The structure of apo tryptophanase from Escherichia coli reveals a wide-open conformation. Acta Crystallogr D Biol Crystallogr 63:969-974. https://doi.org/10.1107/S0907444907036396.

58. Blattner FR, Plunkett G III, Bloch CA, Perna NT, Burland V, Riley M, ColladoVides J, Glasner JD, Rode CK, Mayhew GF, Gregor J, Davis NW, Kirkpatrick HA, Goeden MA, Rose DJ, Mau B, Shao Y. 1997. The complete genome sequence of Escherichia coli K-12. Science 277:1453-1462. https://doi .org/10.1126/science.277.5331.1453.

59. Amann E, Ochs B, Abel KJ. 1988. Tightly regulated tac promoter vectors useful for the expression of unfused and fused proteins in Escherichia coli. Gene 69:301-315. https://doi.org/10.1016/0378-1119(88)90440-4.

60. R Core Team. 2020. R: a language and environment for statistical computing. R Foundation for Statistical Computing, Vienna, Austria. https://www .R-project.org/. 